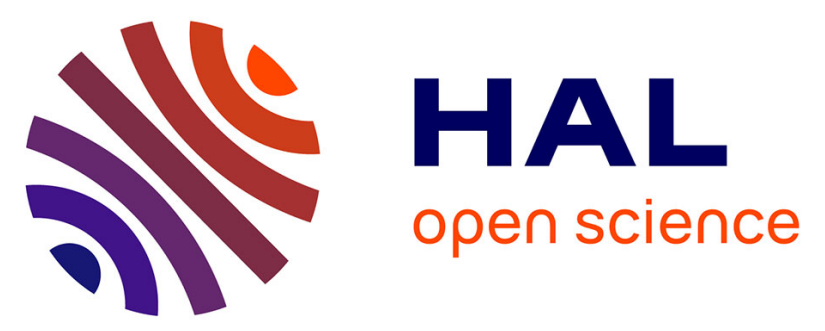

\title{
Acoustic monitoring of a thermo-mechanical test simulating withdrawal in a gas storage salt cavern
}

Cyrille Balland, Joel Billiotte, Bruno Tessier, Anne Raingeard, Emmanuel

Hertz, Grégoire Hévin, Daniel Tribout, Nicolas Thelier, Faouzi Hadj-Hassen, Yvan Charnavel, et al.

\section{To cite this version:}

Cyrille Balland, Joel Billiotte, Bruno Tessier, Anne Raingeard, Emmanuel Hertz, et al.. Acoustic monitoring of a thermo-mechanical test simulating withdrawal in a gas storage salt cavern. International Journal of Rock Mechanics and Mining Sciences, 2018, 111, pp.21-32. 10.1016/j.ijrmms.2018.07.023 . hal-02109136

\section{HAL Id: hal-02109136 \\ https: / hal-mines-paristech.archives-ouvertes.fr/hal-02109136}

Submitted on 3 Aug 2021

HAL is a multi-disciplinary open access archive for the deposit and dissemination of scientific research documents, whether they are published or not. The documents may come from teaching and research institutions in France or abroad, or from public or private research centers.
L'archive ouverte pluridisciplinaire HAL, est destinée au dépôt et à la diffusion de documents scientifiques de niveau recherche, publiés ou non, émanant des établissements d'enseignement et de recherche français ou étrangers, des laboratoires publics ou privés. 
Acoustic monitoring of a thermo-mechanical test simulating withdrawing in a gas storage salt cavern

Cyrille Balland ${ }^{\mathrm{a}}$, Joël Billiotte ${ }^{\mathrm{b}}$, Bruno Tessier $^{\mathrm{b}}$, Anne Raingeard ${ }^{\mathrm{a}}$, Emmanuel Hertz $^{c}$, Grégoire Hévin ${ }^{d}$, Daniel Triboutc ${ }^{c}$ Nicolas Thelier ${ }^{c}$, Faouzi Hadj-Hassen ${ }^{b}$, Yvan Charnaveld and Pascal Bigarréa

alneris, bMines Paritech, 'Salins du Midi, dStorengy 


\section{ABSTRACT}

The natural gas storage in salt caverns requires fast injection / withdrawal cycles due to the increasing dynamics of the energy market. High rates induce rapid changes in the internal pressure of the stored gas causing important temperature changes susceptible to damage the rock salt mass. To experimentally observe this, the Starfish project led to initiate and characterize the damage caused by purely thermal stresses at the surface of a large bloc of rock in the salt Mine of Varangéville (France). The objective was to determine the type of failure mechanism involved with repeated cooling stages. Since the salt is favourable to the generation of Acoustic Emissions (AE) and the propagation of the stress waves, acoustic monitoring has been chosen as one of the methods to follow the impact of the salt cooling. In addition to thermal and mechanical sensors, an acoustic monitoring device consisting of 16 ultrasonic sensors has been installed on the free surface and in boreholes. It enabled to record and locate a large number of $A E(58,426)$ located with good accuracy $(2.5 \mathrm{~cm})$. Those AE can be correlated to the evolution of salt fracturing. Acoustic activity is very intense at the start of each cooling cycle, then it decreases with time to reach a very low level (background) after about 15 days. The average localisation depth reached by the $\mathrm{AE}$ is about $90 \mathrm{~cm}$ during the first cooling period. For subsequent cooling cycles, this depth is limited to $74 \mathrm{~cm}$. All these results show that the first cooling period is decisive, as it contains the strongest and deepest acoustic emissions. It would have been useful to know whether a first cycle with a lower temperature amplitude could have decreased the maximum amplitude and final depth of the AE. This crescendo approach would be useful for operators.

Key words: Acoustic monitoring, Underground salt mine, Gas Storage, Energy, Rock Mechanics, France 


\section{INTRODUCTION}

Natural gas storage in salt caverns requires increasingly rapid injection / withdrawal cycles due to the new dynamic energy market. These cycles are accompanied by rapid fluctuations in the pressure of the stored gas, and then by major temperature variations that may damage the salt cavern. This type of thermal damage due to hot exhaust gases has been observed in the case of a granite formation [1]. Several theoretical studies have been carried out to estimate the amplitude of induced damages in the salt [2] [3]. In order to observe this damage in situ, the SMRI (Solution Mining Research Institute) selected the Starfish project led by Storengy in partnership with Mines-ParisTech, Ineris and Salins du Midi. The purpose of this project was to cool down a salt block to reproduce the thermal solicitations applied to the surface of a salt cavern. The main objective was to initiate and describe the damage caused to the surface of the deposit by stresses of a purely thermal origin. It involved finding out the type of fracture mechanism that occurred and the nature and extent of the fractures. It also involved highlighting possible regression or amplification phenomena damaging the rock salt with repeated cooling cycles.

The study was carried out in partnership between the parties involved in the project. Excavation and development of the mine gallery were performed by Salins du Midi, designing and setting up the cooling system, the visual monitoring and part of the geotechnical monitoring by Mines-Paristech, and a second part of the geotechnical and acoustic monitoring by Ineris. This last part is presented in this paper. 
Acoustic monitoring of the rock damage has been used several times during mechanical tests. King et al. [4] highlighted a correlation in the laboratory between acoustic emissions ( $\mathrm{AE}$ ) and fracture density during triaxial tests on sandstone. Dahm [5] and Moriya et al. [6] studied acoustic activity in the salt. They demonstrated that this material shows an intense acoustic activity under mechanical loading with dominant shear mechanisms and minor attenuation of the mechanical waves due to its crystalline structure. Acoustic emissions of thermal origin were studied essentially during warming phases that exceeded the thermal amplitude expected in gas storage: $550^{\circ} \mathrm{C}$ in salt for Dahm [7], $200^{\circ} \mathrm{C}$ for Vasin et al in marble and sandstone [8] or $800^{\circ} \mathrm{C}$ in concrete for Tang [9]. Dahm and Vasin indirectly studied $A E$ during cooling phases after preliminary warming. They demonstrated that acoustic activity reached a peak when cooling began, i.e. when the heat source was cut off. However, this comparison is narrow-limited, as the damage mainly occurs during the warming phase. The Starfish experiments could have followed a similar, and hence better-known protocol. Instead of this, the focus of the experiment was to control the cooling phase to analyse the damage due to cooling. Indeed, thermal contraction of the rock occurs, which can lead to the onset of tensile stresses which can exceed salt resistance (around 1-2MPa) and therefore initiate damage. Warming can also generate damage with shear stresses (spalling). However, it is necessary to have larger thermal amplitudes than for cooling to exceed the salt resistance. This mode of damage is not observed in this study.

As salt is a favourable medium for the generation of $A E$ and the propagation of their signals, the acoustic monitoring method was chosen for monitoring the cooling phases. It is expected that this method will enable to quantify and locate the damage in the stressed deposit. Several special methods were used in this study for locating acoustic emissions and estimating their magnitude and mechanisms. The implementation of the experiment and the results obtained are set out in this paper. 


\section{EXPERIMENTAL DESIGN}

\subsection{EXPERIMENTAL SETTING}

The experimental site is located in a blind gallery in the Varangéville salt mine excavated for this purpose by the operator, Salins du Midi (Figure 1). The experiment covers a $10 \mathrm{~m}^{2}$ horizontal area of the salt deposit at the most homogeneous location in the gallery. In particular, the experiment had to take place at a distance from the walls and to avoid the drying cracks (dotted line on the Figure 1) that formed at the time when the salt was deposited to have an homogenous rock volume. To perform the test, a $13 \times 12 \times 1.3 \mathrm{~m}^{3}$ slab was left unexcavated at the floor of the gallery. The distance from the top surface of the salt block to the salt-marl contact is about $1.5 \mathrm{~m}$. In order to isolate the effects of a rapid cooling on the stress state, the influence of the in situ stress field was minimized. For this purpose, two vertical slots were cut parallel to the niche axis, at $1.1 \mathrm{~m}$ and $0.7 \mathrm{~m}$ from the West and East walls, respectively. The slots were 0.1 $\mathrm{m}$ wide and $1.5 \mathrm{~m}$ high, and were backfilled prior to the test to prevent heat convection. Thanks to the slots, the salt block is free at four surfaces: top, front and two sides.

This salt (94\%) is mineralised on a macroscopic level with centimetre-sized crystals. Its mean density is 2.2. The Young's and Poisson's moduli of the salt are $25 \mathrm{GPa}$ and 0.25 respectively, with a uniaxial compressive strength of $30 \mathrm{MPa}$ and a tensile strength of 1 to $3 \mathrm{MPa}$. Its thermal expansion coefficient is $3.8 .10^{-5} /{ }^{\circ} \mathrm{C}$. For the rest of the study, the tensile stress-strain values are negative by convention. 
A cold chamber was installed by Armines [10] over the prepared area. The cold chamber comprises five insulated faces with two access doors (north and south faces); the evaporator of the cooling system was fixed on the ceiling along the east side and a ventilator unit was set up on the floor along the west side. The chamber was designed so that a temperature drop of $40^{\circ} \mathrm{C}$ could be applied to the salt surface (from the ambient temperature to about $-25{ }^{\circ} \mathrm{C}$ ). The mine is ventilated, and temperature is nearly constant in the drifts $\left(14.5^{\circ} \mathrm{C}\right.$ approximately at $120 \mathrm{~m}$ depth, according to the geothermal gradient). To ensure a uniform cold production inside the chamber, the cold air was blown through an evaporating system placed in the ceiling along the right (East) wall of the chamber.

The salt deposit was subjected to 4 cooling cycles with a set point temperature in the cooling chamber of $-9{ }^{\circ} \mathrm{C}$ over a minimum period of 28 days for the first 3 cycles. The main purpose was to compare how the damage develops as the cooling cycles progress. The warming phase between cooling cycles varies (from 1 month between the $1^{\text {st }}$ and $2^{\text {nd }}$ cycle to 2 months between the $2^{\text {nd }}$ and the $3^{\text {rd }}$ cycle) (Figure 2) as it is not controlled and is accompanied by substantial condensation which required the surface and the sensors to be systematically reconditioned. The last cooling phase, not planned in the experiment, took place in two stages, the first one lasted 14 days only at $-9^{\circ} \mathrm{C}$ and the second one 22 days at $-27^{\circ} \mathrm{C}$. The main purpose of this last cycle was to monitor the damage under increased stress. 


\subsection{INSTRUMENTAL SETTING}

An extensive measurement program was defined to get as much insight as possible. 26 temperature sensors (Pt100 and thermocouples) were installed at different locations in and out of the chamber. The heat flux between the air and the salt surface was also measured, using a flux sensor placed on the floor, in the central part of the chamber. Strain gauges were placed on the salt surface and in the central borehole. An imaging system was also set up; it allowed tracking the formation and propagation of possible fractures. 4 digital cameras (resolution of 3 $\mathrm{px} / \mathrm{mm}$ ) fixed on the ceiling covered the entire floor of the chamber. A rotating infrared camera was also placed at the ceiling to track the chamber surface temperature. All these techniques allowed a full characterization of possible fractures: time of creation, location and orientation, size, number, etc.

in addition, the ultrasonic monitoring system comprises $16 \mathrm{R} 6 \mathrm{\alpha}$ piezoelectric sensors (peak frequency of the equivalent instrumental response centred on 60 $\mathrm{kHz}$ ) arranged either on the surface, adhering to the salt deposit or embedded into 5 boreholes (Figure 3). 11 sensors are used as receivers (named R1 to R11) and the 5 others as transmitters (E1 to E5) to know the velocity field of the salt block. The transmitters are connected to a special box that can emit 500-volt pulses, while the receivers are connected to an Hyperion acquisition device with a maximum $5-\mathrm{MHz}$ sampling frequency per channel. .

The ultrasonic sounding array is centered on the nude floor of the cold chamber in order to monitor the most homogeneous section. The purpose was to avoid potential edge effects due to the ventilators, walls and doors. Receivers R5, $\mathrm{R} 6, \mathrm{R} 7$ and $\mathrm{R} 8$ are laid out on the surface at the four corners of a $2 \mathrm{~m} \times 2 \mathrm{~m}$ square (Figure 4). The four transmitters E1, E2, E3 and E4 are located at the middle of the sides of this square. Transmitter E5 and receiver R9 are placed together on the surface at its center. Receivers R1, R2, R3 and R4 are installedd at the bottom of four inclined 60-cm-deep at boreholes located at about $70 \mathrm{~cm}$ from the center of the monitored square. Receivers R10 and R11 are located at 20 and $40 \mathrm{~cm}$ respectively below the salt surface into a vertical central borehole. 
The surface sensors are glued facing downwards. The two sensors in the borehole are sealed facing horizontally towards sensor E2, while each of the oblique borehole sensors faces the top of the borehole perpendicular to the boreholes $\left(25^{\circ}\right)$, i.e. inclined at about $65^{\circ}$ on the axis of each borehole. Note that the surface of the deposit is slightly inclined towards the north, which results in a difference in altitude of $13 \mathrm{~cm}$ on the monitored area and $9 \mathrm{~cm}$ between sensors $\mathrm{R} 6$ and $\mathrm{R} 8$.

It should be noted that the acoustic activity recorded by the monitoring array closely depends on the acquisition parameters (triggering, frequency...). However, these parameters could not be calibrated without damaging the rock. They were therefore optimised during the first cooling cycle. Acquisition was initially performed with a5-MHz sampling frequency with relatively low triggering threshold (500 mV for a noise level inferior to $100 \mathrm{mV}$ ) and a minimum of 3 triggering channels. After 143 minutes during the first cooling phase, the number of recorded AE exceeded the memory capacity of the acquisition system reaching a maximum of 6000 recorded events. As a result, the triggering threshold and the minimum number of triggering channels were repeatedly raised up while the sampling frequency was reduced to $2.5 \mathrm{MHz}$ for signals with a maximum $150-\mathrm{kHz}$ frequency content.

The acquisition parameters chosen for the following cooling cycles were identical to those at the start of the first cycle in order to allow easier comparison in the acoustic activity rate. Only the sampling frequency was fixed at $2.5 \mathrm{MHz}$. Since there was a lower $A E$ rates during cycles 2, 3 and 4, the system memory was not saturated again as during the first cycle. The period of the first cycle that could be compared with the others therefore corresponds to the first 143 minutes. For this reason, this period was chosen for strict comparisons between cycles later in the study.

A series of active stacked emissions was performed at midnight every 24 hours from the 5 transmitters towards the receivers with the same acquisition parameters as for passive acoustic monitoring. The rate was adjusted to a minimum in order to disrupt the acoustic monitoring as little as possible and also because of the small expected variations in the velocity field. 
Both passive and active acoustic monitoring were set up during the cooling periods. During the natural and forced warming phases, the acoustic sensors were deactivated due to the appearance of water condensation on the surface of the salt, which led to short-cut of the gauges and decoupling the acoustic sensors. This water evaporated naturally by the end of the warming phase. This is why only the cooling periods are studied here.

\section{PROCESSING METHODS}

\subsection{LOCATING THE ACOUSTIC EMISSIONS}

Acoustic sources are located in the space-time domain from the arrival times measured at the 11 receivers. Considering the amount of data (several tens of thousands of $\mathrm{AE}$ ), picking the whole arrival times manually proved impossible. They are therefore automatically picked using a ratio which evaluates the ultrasonic signal level on two sliding temporal windows. Arrival time is picked when this ratio exceeds a threshold adapted to the acoustic amplitude level and to the incident wave. However, signals can be erroneously picked, particularly if another signal is superimposed on the same recording, if the signal offset is wrongly established or if the noise level is too loud. The threshold was thus optimised between two thresholds that were too low, causing picking too early, and thresholds that were too high to ensure triggering of most events.

Best ray path solution from one point to another in the medium is then calculated using the Fast Marching Method (FMM) [11] . The location parameters were adjusted thanks to the emission of a calibrated signal from each of the 5 piezoelectric transmitters in the monitoring array, whose positions are precisely known, with an average P-wave velocity calculated at $4457 \mathrm{~m} / \mathrm{s}$ and a standard deviation of $79 \mathrm{~m} / \mathrm{s}$. In order to compare the different localisation parameters, the $X, Y$ and $Z$ differences between the calculated location and the known location of the transmitters were calculated for each of the 5 emission tests. Location deviation was thus obtained with the following formula: 
$\mathrm{dl}$

$$
\begin{aligned}
& =\sqrt{\left(X_{\text {mesured }}-X_{\text {transmitter }}\right)^{2}+\left(Y_{\text {mesured }}-Y_{\text {transmitter }}\right)^{2}+\left(Z_{\text {mesured }}-Z_{\text {transmitter }}\right)^{2}} \\
& =\sqrt{d X^{2}+d Y^{2}+d Z^{2}}
\end{aligned}
$$

The average of the location deviation calculated from the 5 tests was chosen as an indicator to quantify the localisation quality. The lower the average value, the better the parameters selected for localisation.

$\mathrm{AE}$ localisation search area is forced within a $3.8 \times 3.8 \times 1.25 \mathrm{~m}^{3}$ volume, i.e. centred on the middle of the cooling chamber (X: $1.9 \mathrm{~m}, \mathrm{Y}: 1.9 \mathrm{~m})$ and extending over $1.20 \mathrm{~m}$ under the average altitude of the surface and $5 \mathrm{~cm}$ above it. Extending the search area over the zero level on $Z$ axis is required to take into account the ground irregularities. This also enables to eliminate events considered as wrongly located or external to the chamber, in particular those that cluster at the extreme values of the area. Thus events that take place at an actually greater depth or events that are wrongly localised due to incorrect picking are numerically "blocked" against the edges of the area. Events blocked $5 \mathrm{~cm}$ above the virtual ground surface are clearly wrongly localised.

The accuracy of the space distribution of the acoustic sources is improved by discretizing of the search grid from 15 to $1 \mathrm{~cm}$. In addition, all the receivers from which data are picked must be considered, including sensor R9. Although it gives a major misfit for numerous $A E$, because of its central position in the examined area, it actually also constrains the depth of the location.

\subsection{MAGNITUDE DETERMINATION}

The ultrasonic sensors used for the experiments are resonant. Therefore they do not give the physical value that could make it possible to define a local magnitude scale. An experiment has been developed to mitigate this shortcoming. It involves dropping a ball at a known location. The impact energy of the ball can be deduced from the height from which it is dropped. Repeating this operation at different heights gives enough data to determine the dimensionless unknowns: $\kappa$, $\beta$ and $\gamma$. which are defined in the relationship linking the source energy $\mathrm{E}_{\text {source }}$ and the sensor energy $E_{\text {sensor }}$ (in J) [12]: 


$$
E_{\text {source }}=\left(\frac{E_{\text {sensor }}}{\kappa D^{\beta}}\right)^{1 / \gamma}
$$

where $D$ is the distance between the sensor and the source.

The factor $\kappa$ corrects the resonance; the source energy values obtained are only apparent. To this purpose, the following equipment is required: a steel ball with a known mass $\mathrm{m}$, a video camera to record the trajectory of the ball, particularly making an accurate observation of the drop height and any rebounds, and a ruler to be used as a scale on the video recording. The free-falling ball has a constant drop energy whose value is determined by:

$\mathrm{E}_{\text {drop }}=\mathrm{E}_{\text {kinetic }}+\mathrm{E}_{\text {weight potential }}=\frac{1}{2} \mathrm{mv}^{2}+\mathrm{mgh}$

where at any time: $\mathrm{m}$ is the mass of the ball $(\mathrm{kg}), \mathrm{g}$ is the acceleration of the weight $(9.81 \mathrm{~N} / \mathrm{kg}), v$ is the velocity of the ball $(\mathrm{m} / \mathrm{s}), \mathrm{h}$ is the height of the ball $(\mathrm{m})$ and $E$ the energy values $(J)$. Given that the ball was released at a speed of zero and from the height $h_{1}$ :

$\mathrm{E}_{\mathrm{drop}}=\mathrm{mgh}_{1}$

The dropping location of the ball was chosen near the centre of the ultrasonic array and the camera was placed in a position from which the entire drop and the rebounds could be filmed (Figure 5). The dropping ball was filmed, selecting different release heights varying from 10 to 100 centimetres in $10-\mathrm{cm}$ steps. The experiment was repeated several times in order to record the acoustic signals corresponding to the impact and to select the clearest rebounds. The signal period corresponding to the initial drop had to be selected so as to measure the energy at the sensor for this part of the signal only. So, the beginning and the end of each event was marked for each of the 11 signals at the receivers with the same duration. 
Distance is calculated between the impact and the 11 receivers for each test, which provides thus 110 values. For each test, height $h_{1}$ had to be accurately determined using the video recording of the drop as well as the theoretical source energy $E_{\text {source-theo. }}$ The energy at the sensor was then determined at the time of the first impact, i.e. by picking the beginning and the end of the signal corresponding to the first drop. 110 values were hence retrieved to determine the source energy, using the previously calculated parameters $k, \beta$ and $\gamma$. These are normally equivalent to the level of all the sensors for one and the same event. The parameters were then optimised by the least squares method, minimising the sum of the residues between the theoretical source energy (calculated from relation (4)) and the average of source energy values measured by the different sensors:

$\sum_{\mathrm{i}=1}^{10=\text { number of drops }}\left(\frac{1}{11}\left(\sum_{\mathrm{j}=1}^{11}=\text { number of sensors } \mathrm{E}_{\text {source }_{\mathrm{ij}}}\right)-\mathrm{E}_{\text {source-theo }_{\mathrm{i}}}\right)^{2}$

Finally, the system consists of 110 equations with 3 unknowns. Solutions were calculated using an algorithm named GRG (Generalised Reduced Gradient). This type of equations system can provide local minima, i.e. several solutions depending on the initial values. The results is confirmed by testing different initial values of the unknowns. The relationship between the source energy and the sensor energy then gives $\mathrm{K}=12700.47, \beta=-0.001826417$ and $\mathrm{y}=0.7482615$.

Using the energy measured at the sensors for each test, the source energy and the magnitude are calculated using the Gutenberg-Richter formula [13] and traditional parameters of the following type:

$\log \left(E_{\text {source }}\right)=1.5 \mathrm{Mag}+11.8$ 
For the ball drop tests, the magnitudes of the tests vary between -4.7 and 3.4 with a power distribution depending on the drop height. Considering the source energy as measured by all sensors one by one, the values are scattered, particularly for greater heights. This is probably due to anelastic energy losses (stress-strain, friction, etc.) and to the dominant surface waves, in particular for the sensors adhering to the surface. When the average source energy for each test is calculated from the values of the 11 sensors, the calculated source energy is in line with the theoretical one, which was calculated using: $E_{\text {source }}=m g h_{l}$. This results in a correlation coefficient exceeding 0.97 (Figure 6). For the next part of the study, this method was used to estimate the magnitude of the acoustic emissions.

\subsection{Determining THE SOURCE MECHANISMS}

$\mathrm{AE}$ are transient elastic waves generated by a rapid release of energy in a medium. They occur when micro-fractures rapidly grow in the media such as native salt. One of the purposes of the study is to describe the source mechanisms of the fracture responsible for the AE. Ohno and Ohtsu [14] developed a method for describing fractures in concrete. This method only uses relative amplitude values adequate to resonant acoustic sensors, unlike moment tensor inversion methods or those using the direction of the first motion.

Two factors are used for this parameter study. The first one is an average frequency, which can be calculated from the ratio of the number of occurrences the threshold is exceeded over the duration of an AE. The second one is a value named RA, which corresponds to the ratio of the signal rise time over the maximum amplitude (the rise time corresponds to the time elapsed between the moment when the threshold is first exceeded to the maximum amplitude peak).

Traction events make the edges of the fracture moving apart, which results in a volume change of the medium. In consequence, most of the energy is transmitted in the form of longitudinal or $\mathrm{P}$ waves (Figure 7), while the slower $\mathrm{S}$ waves are less energetic or even non-existent. This means that most of the energy is registered early; the signal rise time is fairly short, resulting in a large wave angle. 
On the other hand, a shear stress event makes the shape of the medium change near the fracture, but not its volume, thereby shifting the greater part of the energy towards shear waves, or S waves. The maximum amplitude occurs some considerable time after the moment when the threshold is first exceeded and the rise time is longer. To validate this method in our study, two manual $A E$ were generated, one following the same model as the ball drops, representing a tensile/compressive $\mathrm{AE}$, and the other involving sticking a piece of salt to the surface with phenyl salicylate, which is instantly broken off by a shear stress. The maximum signal energy arrives after $4.2 .10^{-4} \mathrm{~s}$ for the shear stress and only $1.6 .10^{-3} \mathrm{~s}$ for the tensile mechanism. Moreover, tension events are characterised by a higher frequency. As a result, the events can be easily described by comparing the two factors (average frequency and RA): when the average frequency of an event is represented as a function of the RA value, the mechanism can be described as tending towards a tension or shear mechanism. It therefore appears that a parameter analysis is applicable to the study of the experimental mechanisms. This is even more certain if the "reference" signals have known mechanisms and can be used to define the relationship between RA and average frequency in order to move from one mechanism to another.

\section{RESULTS}

\subsection{ACOUSTIC ACTIVITY}

During the four cooling cycles, 58426 AE were recorded. The number of recorded $A E$ was the largest for the first cooling cycle, with 21614 emissions, and the lowest for the third cooling cycle, with 8124 emissions. The number of AE would probably have been considerably greater during the first cooling cycle if the acquisition system had not reached its saturation level. By extrapolating the first 143 minutes, the total number of $\mathrm{AE}$ in the first cooling cycle would approach 47 000 to 60000 , depending on whether the second or the third cooling cycle is used for extrapolation.

In Figure 8 , the $4^{\text {th }}$ cooling cycle has been split into two cooling stages, 4' and 4", so as to be able to extract the first stage at $-9^{\circ} \mathrm{C}$ identical to the 3 first cooling cycles. The total number of $\mathrm{AE}$ is nevertheless not comparable over the 
entire period, as the cooling cycle only lasted half as the time of the 3 others, i.e. 14 days. Cooling cycle 4' can nevertheless be compared to the other cooling cycles over the first twelve hours for cycles 2 and 3, and over the first 143 minutes for cycles 1,2 and 3. These two periods show that the activity increased in the $4^{\text {th }}$ cooling cycle at an intermediate level between cooling cycles 2 and 3 . Finally, the general trend of the activity follows a reducing power law. This law was calculated from the first 143 minutes and is of the following type: Number of $A E$ in the $n^{\text {th }}$ cooling cycle $=12878 \mathrm{n}^{-1.296}$. During the cooling period of all cycles, the activity was strongest at the beginning (Figure 9): more than $54 \%$ of the $A E$ already occurred during the first 12 hours of cycles 2 and 3 . After 3 days, almost $80 \%$ of the AE had occurred. The trend is the same for cooling cycle 4', although the overall duration is shorter. The shape of the curve for cooling cycle $4^{\prime \prime}$ at $-27^{\circ} \mathrm{C}$ is different: less $A E$ are recorded at the beginning; and after 12 hours, only $27 \%$ of the them have been produced. This difference should be viewed considering that the temperature decreased more slowly down to $-27^{\circ} \mathrm{C}$ set point instead of $-9^{\circ} \mathrm{C}$ as it was set in the previous cycles.

Focusing on the curves showing changes in the number of $A E$ during the first 143 minutes of the 4 cooling cycles, the rate of acoustic activity (the number of AE per unit of time) reaches a maximum only 5 to 7 minutes after the start of the cooling cycles and increasingly rapidly decreases in each subsequent cycle. This happens very slowly after about an hour in the first cycle ( 0.040 day); after 36 minutes in the $2^{\text {nd }}$ cycle $(0.025$ day) and after about 17 minutes in the 3rd and 4th cycles ( 0.012 day). The acoustic activity rate at the start of the $4^{\text {th }}$ cycle is greater than for the $2^{\text {nd }}$ cycle because it drops more quickly with a lower final number of $\mathrm{AE}$.

\subsection{ACOUSTIC ENERGY}

The empirical method set out above was used to estimate the magnitude of all localised AE. It varied between -8 and -3 with the vast majority being between 5.8 and -3.5. The magnitude of $A E$ includes that of the pulses and the ball drops. As calibration is based on the ball drops, the quality of the magnitude estimate is probably better within their range, i.e. between -5.1 and 3.8 (Figure 6). It should also be recalled that the $\mathrm{AE}$ with the highest magnitudes have one or more 
saturated signals. Their actual magnitude could therefore be slightly underestimated.

With regard to the cooling cycles, the most remarkable observation is the much higher magnitude measured for the $1^{\text {st }}$ cycle, with a difference of almost 0.5 compared to the AE dominating the $2^{\text {nd }}$ cycle (Figure 10). The first cycle AE cover the same range of magnitude as for the other cycles, but they are distinctly more numerous, particularly above -4.5 . More precisely, the sharp drop in magnitudes continues, but more moderately in the subsequent cycles. Finally, the $\mathrm{AE}$ at the beginning of the last cooling cycle at $-9^{\circ} \mathrm{C}\left(4^{\text {th }}\right)$ have the lowest magnitudes. The average magnitude of $A E$ then rises with the $-27^{\circ} \mathrm{C}$ stage, but the thermal loads are no longer comparable.

Another classical approach to describe the magnitude is the GutenbergRichter relationship [15] between the number of events $(N)$ and the magnitude (M) $(\log N=a+b M)$. The constant $a$ is a measurement of the level of seismicity and the constant $b$ (or b-value) describes the relative size distribution. In our case (Figure 11), the $b$-value is estimated for the magnitude between -5.2 to -4.0 considering 6 different depth zones of locations of the AE. The results show a clear difference between the $b$-value of the $1^{\text {st }}$ cooling and the other ones. For the $1^{\text {st }}$ cooling, the b-value is low near the surface (0.8) and decreases with depth to 0.6. For the other cooling periods at shallow surface, the b-value is higher (over 1.0), it increases to the middle depth to $1.4-1.5$ and then slowly decreases to the deeper zone. 


\subsection{SPATIO-TEMPORAL DISTRIBUTION OF ACOUSTIC EMISSIONS}

The distribution of this acoustic activity was analyzed by considering the level of AE. For this purpose, all AE with at least 10 picked signals were selected and localized. This corresponds to $40490 \mathrm{AE}$ out of a total of 58426 , i.e. $69 \%$. The uncertainty of the localisation of the pulses is $2.5 \mathrm{~cm}$ and is probably of the same order for the $\mathrm{AE}$ or even better, as the signals of $\mathrm{AE}$ are stronger than the pulses summed up 100 times. The first arrival of these selected AE is therefore better defined than that of the pulses with efficient automatic picking. A manual checking performed on a sampling of signals showed that the automatic picking was occasionally poor on one or two signals when the AE had a low signal to noise ratio. The global ratio of poor localizations is estimated at $5 \%$. Localization results are therefore more accurate for stronger $\mathrm{AE}$, with a high signal to noise ratio.

During the $1^{\text {st }}$ hour, most of the $A E$ were in a $20-\mathrm{cm}$ thick surface strip. During the $2^{\text {nd }}$ hour, the location depth of most of the $A E$ appears to increase, with a more pronounced effect in the left half of the array close to the ventilators, constituting a cluster along a slightly inclined dip, equal to $\sim 2^{\circ}$ for cycle 1 , toward the ventilators (toward the Western side). As the cycles progress, the densest central part of the cluster also tends to move toward the Eastern side.

Estimating the depth of the $A E$ over a moving average window (up to 100 $A E)$ versus time for each of the cycles (Figure 12), makes the increase in depth appear more clearly: with time, the $A E$ follow each other in time at widely scattered depths. However, with a moving average of $10 \mathrm{AE}$, then of $100 \mathrm{AE}$, the average depth of each cycle follows a relatively uniform curve towards a final depth of -0.90 $\mathrm{m},-0.74 \mathrm{~m},-0.71 \mathrm{~m}$ and $-0.72 \mathrm{~m}$ respectively for cycles $1,2,3$ and 4 . Cycle $1 \mathrm{AE}$ thus propagate at a greater depth than those from the other cycles which exhibit a common final depth. This difference is mainly concentrated in the first 143 minutes of the first cooling cycle. Globally, the vertical depth migration velocity of the AE cluster at the beginning of the cooling cycle is estimated at around 8 to $10 \mathrm{~cm} /$ hour depending on the cycle. 


\subsection{STRESSES AND DAMAGE MECHANISMS}

\subsubsection{SCALE OF ACOUSTIC SOURCES/FRACTURES}

Thermal fields are comparable between one cycle and another; the temperature curves, at the surface as well as in boreholes, show the same time and amplitude variations. The acoustic activity variations from one cycle to another are an evidence of the progressive damages into the medium. However, acoustic activity is filtered by the range of sensors in the array, typically between $20 \mathrm{kHz}$ and $120 \mathrm{kHz}$. In other words, the array acts as a filter that only registers the $\mathrm{AE}$ that correspond to fractures radiating from waves within this frequency range.

According to the Goodfellow and Young classification [16] and to the Brune model [17] extended to sources of tensile stress, with a dominant magnitude between -5.8 and -3.5 , the approximate radius of the sources or mechanical fractures is $5 \mathrm{~mm}$ for the smallest and $15 \mathrm{~cm}$ for the largest. As the dominant magnitude is about -5 (Figure 13), the size of most sources is therefore comprised between 2 to $3 \mathrm{~cm}$. This characteristic size is compatible with the network of fractures observed at the surface of the deposits corresponding to cracking around the salt crystals.

\subsubsection{DYNAMICS OF THE COLD FRONT AND DAMAGE}

It is possible to monitor the propagation of the cold front through the medium by measuring the temperature gradient (as a function of depth) between the 4 borehole temperature probes (T2, T3, T4 and T5). These gradients are represented during the first cooling days of cycle 2 (Figure 14). The curves for the other cycles are almost identical except when the door opened unexpectedly and provoked defrosting during the $1^{\text {st }}$ cycle. 
Considering the position of the temperature probes, the temperature gradients can be locally calculated at the midpoint between the probes, i.e. at 13$\mathrm{cm}, 33-\mathrm{cm}$ and $62-\mathrm{cm}$ depth for gradients T2-T3, T3-T4 and T4-T5 respectively. These curves show that the gradient is the greatest at $13 \mathrm{~cm}$ for T2-T3 with $31.1^{\circ} \mathrm{C} / \mathrm{m}$ after 2 hours and 45 minutes of cooling. This gradient is likely to be substantially greater and shallow at the very beginning of the cooling cycle. For the measured values, the deeper, the later the maximum value is reached: after 12 hours 35 minutes for gradient T3-T4 and after 25 hours 5 minutes for gradient T4T5.

Figure 14 shows a comparison between the variation of the measured gradients and the depths of the AE. When the gradient at 13-cm depth is at its maximum (after 2 hours 45 minutes or 0.11 day), most of the $A E$ (i.e. the cluster) are located at $30-\mathrm{cm}$ depth approximately during the three cycles 2, 3 and 4.When the gradient at $33-\mathrm{cm}$ depth is at its maximum (after 12 hours 35 minutes or 0.52 day), most of the $A E$ are located at $45-\mathrm{cm}$ depth. When the gradient at $62-\mathrm{cm}$ depth is at its maximum (after 25 hours 5 minutes or 1.05 days), most of the AE are located at $52-\mathrm{cm}$ depth. This shows that the $A E$ and their related fractures are initiated ahead of the front at shallow levels and behind the front at greater depths. The depth of the $A E$ is controlled both by the position of the cold front and by the initial stress field. The medium is increasingly confined with depth. The thermal stress required to initiate new fractures increases with depth. 
The fact that the $\mathrm{AE}$ were deeper during the first cooling cycle is probably also due to this confinement and the higher-energy fractures $(M \sim-4)$. These fractures, which are unique to cycle 1 , of the order of $15 \mathrm{~cm}$ in radius, may thus propagate at a deeper level (down to $90 \mathrm{~cm}$ ) than fractures from subsequent cycles $(70-74 \mathrm{~cm})$. Note that this difference in depth corresponds to the order of magnitude of the radius of the largest recorded fractures. Figure 14 also shows that the depth of the $A E$ is highly sensitive to the temperature gradient. For the depth curve of the $2^{\text {nd }}$ cycle, the depth of $A E$, although averaged over 100 values, shows fluctuations that closely correlate with the gradient variations induced by successive defrosting events. In other words, an increase in surface temperature during defrosting locally increases the average depth of the $A E$ on several centimetres. This may be due to a drop in acoustic activity in the shallowest part, which automatically decreases the average depth of the spread of $A E$. 


\subsubsection{SOURCE MECHANISM}

The Ohno and Ohtsu method previously described and empirically verified was applied to the first and last $A E$ in cycles 2 and 3 . This processing was carried out for the first and the last $A E$ in cycles 2 and 3 (Figure 15). For these 2 cooling cycles, most of the $A E$ were classified in the tension mechanism category at the beginning of the cycles. Note that these fractures at this moment occurred essentially at shallow depths (a few centimetres), near the free surface of the medium. At the end of the cooling period, the AE were classified more in so-called mixed mechanism categories or as shear stress mechanisms. This trend is clearer for the $3^{\text {rd }}$ cooling period, where more than half the $A E$ are in the shear mechanism category.

The AE deepening is the most likely assumption to consider linked to this changing mechanism during the cooling period. Near the surface, tension fractures tend to occur because the salt is less confined, because of the free surface. Before cooling, the floor of the room is submitted to a tangential stress as the vertical component is null (free face). Due to the large span of the chamber $(13 \mathrm{~m})$, the tangential stress corresponds to a tension, but its magnitude does not exceed the salt strength. The creation of the two vertical slots contributes even more to the development of this tension by reducing the confinement effect brought by the initial stresses (isotropic stress regime). At a greater depth, i.e. at about $70 \mathrm{~cm}$ for the two examples set out here, the fractures occur in a more confined medium. This environment thus favours shear mechanisms which can theoretically occur at a constant volume or in any case with much smaller variations in volume than tension mechanisms. This observation can be correlated with numerical modelling by analysing the fracture criteria as a function of depth.

\section{DISCUSSION}

This experiment is very different from other experiments related in the literature, where rock mass is generally heated from its initial state and not cooled, as it is the case here. This experiment can be compared to the experimentation of Dahm [7], just after switching off the heating element in the massive salt mine of Asse (Germany). In his case, the number of AE was more important around where 
the heating probe was located. AE migrated with time deeper into the salt rock than in our experiment. However, in our case, it was the compressive stress which decreased and caused the $\mathrm{AE}$ as we create directly tensile stresses. Although both types of cooling generate $A E$, they do not show the same type of mechanism. Cooling increases the magnitude of the tangential stress which corresponds initially to a low tension as explained. This increase is done rapidly in the first few hours as shown by Blanco-Martin [10]. Fractures are created when the tensile stress exceeds the tensile strength of salt and subsequently $\mathrm{AE}$ are generated. On the contrary, warming leads to the reverse effect: the tensile stress is reduced and the created fractures are closed. In our experiment, the source mechanism is almost always entirely in tension while it was shearing in Dahm's experiment: " The estimated double-couple radiation pattern of observed sources indicates macroscopic shear failure, possibly on preexisting slip planes or zones of weakness. However, we cannot completely rule out that tensile cracks". The acoustic activity is weak for the Varangéville salt warming in comparison to the intense activity in the Asse salt warming. This is probably related to the change in temperature difference of $24{ }^{\circ} \mathrm{C}$ and $500{ }^{\circ} \mathrm{C}$ respectively and the return to equilibrium in one case and a significant disruption in the other case.

Regarding the difference between the first and the following cycles, the total number of $\mathrm{AE}$, the average magnitude and the maximum depth are all three very different. The salt massif, subject to thermal stresses, behaves as if there were two different materials: one before and one after the first cycle. This behavior is typically observed for other types of mechanical processes. Bandis et al [18] observed that the maximum deformation and damage occur at the beginning of the first cycle for a discontinuity under a normal stress. During the following cycles, the deformations are much lower and are quite similar between them. However, these observations relate only to $A E$, at the same time the $P$ wave velocity in the solid showed no significant change (less than the measurement accuracy). As illustrated, the massif is damaged by $\mathrm{AE}$; however, this disturbs little the velocity field and the dynamic mechanical parameters related to this P-wave propagation. This is probably because most of the cracks at the origin of $A E$ are contiguous and ensure overall continuity of salt. 
Another way to describe the cracks is to follow the b-value changes of the $\mathrm{AE}$ with cooling and depth. For the $1^{\text {st }}$ cooling, the b-value decreases with depth as described by Mori and Abercrombie [19]. They explained that the small cracks cannot generate bigger cracks in the shallow surface because the heterogeneity of the rock stops its coalescence and the stresses are not sufficient. "At greater depth, more homogeneous material leads to a greater probability that the rupture can continue to grow into a larger event". In our case for the $1^{\text {st }}$ cooling, the salt rock is undamaged and thus homogenous. The AE size distribution is probably more constrained by the stress field. However, for the other cooling cycles, the rock material is different: its damage in the first cooling undoubtedly forced the size distribution of the AE. The maximum b-value could be an indicator of damage within the rock mass.

This study help to complete numerical models developed by Sicsic and Berest [3]. In their study, where the wall of a salt rock cavity was subjected to thermal stresses, the decimation of small and shallow cracks was followed by larger and deeper cracks. Although the stress field is very different in our case, the pattern of the AE swarms shows that many small-size cracks appear close to the cold front until nearly the maximum depth. We have thus shown that fracturing is global and diffuse into the rock. The model of Sicsic and Berest [3] rather reproduces the pattern of larger cracks, which is rarely observed in our spatially restricted case. Nevertheless, these large cracks are embedded in a layer with diffused damage. This layer could be similar to the one created around deep excavations [20]. Yet note that the thermal origin cracks are essentially vertical.

For gas storage operators, the cracks should be radial to the cavity. They could preferentially occur near the wellbore, where relaxation and gas flow would be most important. They could also be formed around the base of the salt cavities after injection of fresh cold brine (compared to ambient temperature) from the surface in specific operating phases. Estimating the volume of solicited and associated damaged rock during these phases would anticipate possible changes in the porosity of the wall of the salt rock cavity. 


\section{CONCLUSIONS}

The acoustic monitoring system recorded a large number of AE (58 426). These events were localized with a relatively low level of uncertainty $(2.5 \mathrm{~cm})$. This provides a good representation of cracking propagation inside the cooled salt layer. Regrettably the saturation of the acquisition system during the 1st cycle made it difficult to compare with the 3 others. Similarly, the intense condensation of water during the first days of the warming periods prevented the monitoring. However, this acoustic monitoring provides several important results in response to the main questions raised about the nature and the extent of the damage and fatigue inside the medium due to thermal stress: Acoustic activity is very intense at the start of each cooling cycle, then it decreases with time to reach a very low level (background) after about 15 days; this activity decreases asymptotically from one cycle to another in terms of the number of $A E$ and magnitude, with a power law of the following type: Number of $A E$ in the $n^{\text {th }}$ cooling period $=12878 n^{-1.296}$; with the surface temperature imposed at $-9^{\circ} \mathrm{C}$ compared to the $15^{\circ} \mathrm{C}$ natural temperature in the mine, the average depth reached by the $A E$ is about $90 \mathrm{~cm}$ during $1^{\text {st }}$ the cooling period. For subsequent cooling cycles, this depth is limited to $74 \mathrm{~cm}$. Greater depths are reached during the first cycle because of the highest energy value and of the size of the fractures $(15 \mathrm{~cm})$. The propagation of the cluster formed by the $A E$ is quicker than the maximum thermal gradient in the subsurface, and slower at greater depths; this is due to the predominantly fracture mechanism changing from tensile to shearing one. The two stages in the $4^{\text {th }}$ cooling period show that total acoustic activity increases with slower stresses. This phenomenon is probably linked to differential mechanisms in the salt (relaxation and creep), the effect of which could be clarified by numerical modelling.

Most of the AE have a -5 order of magnitude, which corresponds to the characteristic fracture radii of 2 to $3 \mathrm{~cm}$, i.e. the average size of the salt crystals in the medium. This observation thus shows that fracturing in the deposit is mainly diffuse (Figure 16) along the cold propagation front, in line with the surface cracking observed around the salt crystals. All these results show that the first cooling period is decisive, as it contains the strongest, deepest AE. It would have been useful to know whether a first cycle with a lower temperature amplitude could have decreased the maximum amplitude and final depth of the $A E$ to which the 
salt deposit was subjected. This crescendo approach would be useful for an operator. The $-27^{\circ} \mathrm{C}$ stage of the last cycle tends towards this direction, although it was short-lived, with the cooling unit operating at the limit of its maximum power. It would subsequently be useful to compare the thermal gradient variations with the thermal-mechanical models to account more accurately for the position of the spread and the nature of the AE mechanism in relation to the cold front and the state of the current constraints.

Finally, Figure 16 suggests that in addition to diffuse acoustic activity, alignments of $\mathrm{AE}$ could underline the presence of the large fractures observed on the deposit. Dynamic comparison between the $\mathrm{AE}$ and the visual observations could improve our knowledge of the general fracturing picture of the deposit both in terms of time and studied volume.

\section{ACKNOWLEDGEMENTS}

The authors thank the Solution Mining Research Institute for its financial contribution and technical commitment in the success of the project.

\section{REFERENCES}

[1] Lee C, Tsui K, Tsa A. Thermomechanical response of a disposal vault in a high horizontal stress field. In: Proceedings of ISRM Aachen. 1982. p.961-9.

[2] Brouard B, Frangi A, Bérest P. Mechanical stability of a cavern submitted to high-frequency cycles. SMRI Spring Meeting. Galveston; 2011. p. 99-116.

[3] Sicsic P, Bérest $P$. Thermal cracking following a blowout in a gas storage cavern. Int. J. Rock Mech. Min. Sci. 2014;71:320-329.

[4] King MS, Shakeel A, Chaudhry NA. Acoustic wave propagation and permeability in sandstones with systems of aligned cracks. Geological Society. 1997;122:69-85.

[5] Dahm T. Rupture dimensions and rupture processes of fluid-induced microcracks in salt rock. Journal of Volcanology and Geothermal Research. 2001;109:149-162. 
[6] Moriya $\mathrm{H}$, Fujita T, Niitsuma H, Eisenblatter J, Mantheib G. Analysis of fracture propagation behaviour using hydraulically induced acoustic emissions in the Bernburg salt mine, Germany. Int. J. Rock Mech. Min. Sci. 2006;43:49-57.

[7] Dahm T, Manthei G, Eisenblätter J. Relative moment tensors of thermally induced micro-cracks in salt rock. Tectonophysics. 1998;289:61-74.

[8] Vasin RN, Nikitin AN, Lokajicek T, Rudaev V. Acoustic Emission of QuasiIsotropic Rock Samples Initiated by Temperature Gradients. Physics of the Solid Earth. 2006;42:815-823.

[9] Tang SB, Tang CA. Crack propagation and coalescence in quasi-brittle materials at high temperatures. Engineering Fracture Mechanics. 2015;134:04432.

[10] Laura Blanco-Martín L, Rouabhi A, Billiottea J, Hadj-Hassen F, Tessier B, Hévin G, Balland C, Hertz E. Experimental and numerical investigation into rapid cooling of rock salt related to high frequency cycling of storage caverns. Int. J. Rock Mech. Min. Sci. 2018;102:120-130.

[11] Sethian JA. Fast Marching Methods and Level Set Methods for Propagating Interfaces. Computational Fluid Mechanics, Von Karman, Institute Lecture Series; 1998.

[12] Aki K, Richards P. Quantitative Seismology: Theory and Methods. Freeman Co, 1980.

[13] Gutenberg B, Richter CF. Seismicity of the earth and associated phenomena. Princeton University Press, 1949.

[14] Ohno K, Ohtsu M. Crack classification in concrete based on acoustic emission. Construction and Building Materials. 2010;24:2339-2346.

[15] Gutenberg B, Richter CF. Frequency of earthquakes in California. Bulletin. of the Seismological Society of America. 1944;34:185-188.

[16] Goodfellow SD, Young RP. A laboratory acoustic emission experiment under in situ conditions. Geophysical Research Letters. 2014;41:3422-3430.

[17] Walter WR, Brune JN. Spectra of Seismic Radiation from a Tensile Crack. Journal of Geophysical Research. 1993;98:4449-4459.

[18] Bandis SC, Lumsden AC,. Barton NR. Fundamentals of rock joint deformation. Int. J. Rock Mech. Min. Sci. 1983;20:249-268. 
[19] Mori J, Abercrombie RE. Depth dependence of earthquake frequencymagnitude distributions in California: Implications for rupture initiation. Journal of Geophysical Research. 1997;102:2156-2202.

[20] Blümling P, Bernier F, Lebon P, Martin DC. The excavation damaged zone in clay formations time-dependent behaviour and influence on performance assessment. Phys. Chem. Earth. 2007;32:588-599. 
Fig. 1. Localisation of the deposit and position of the cooling chamber in a Varangéville mine gallery (Salins du Midi).

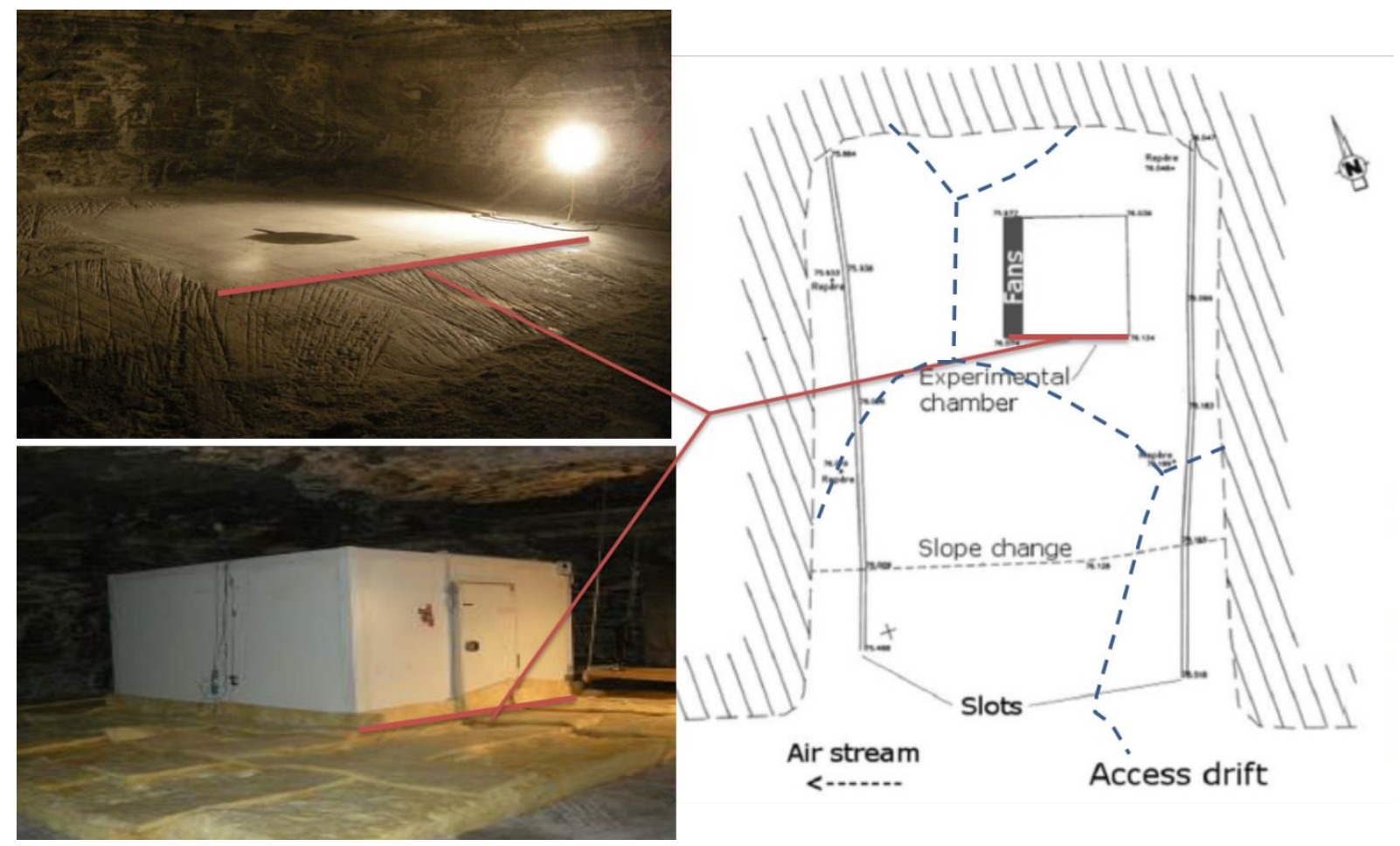

Fig. 2. Distribution of the 4 thermal cycles with time.

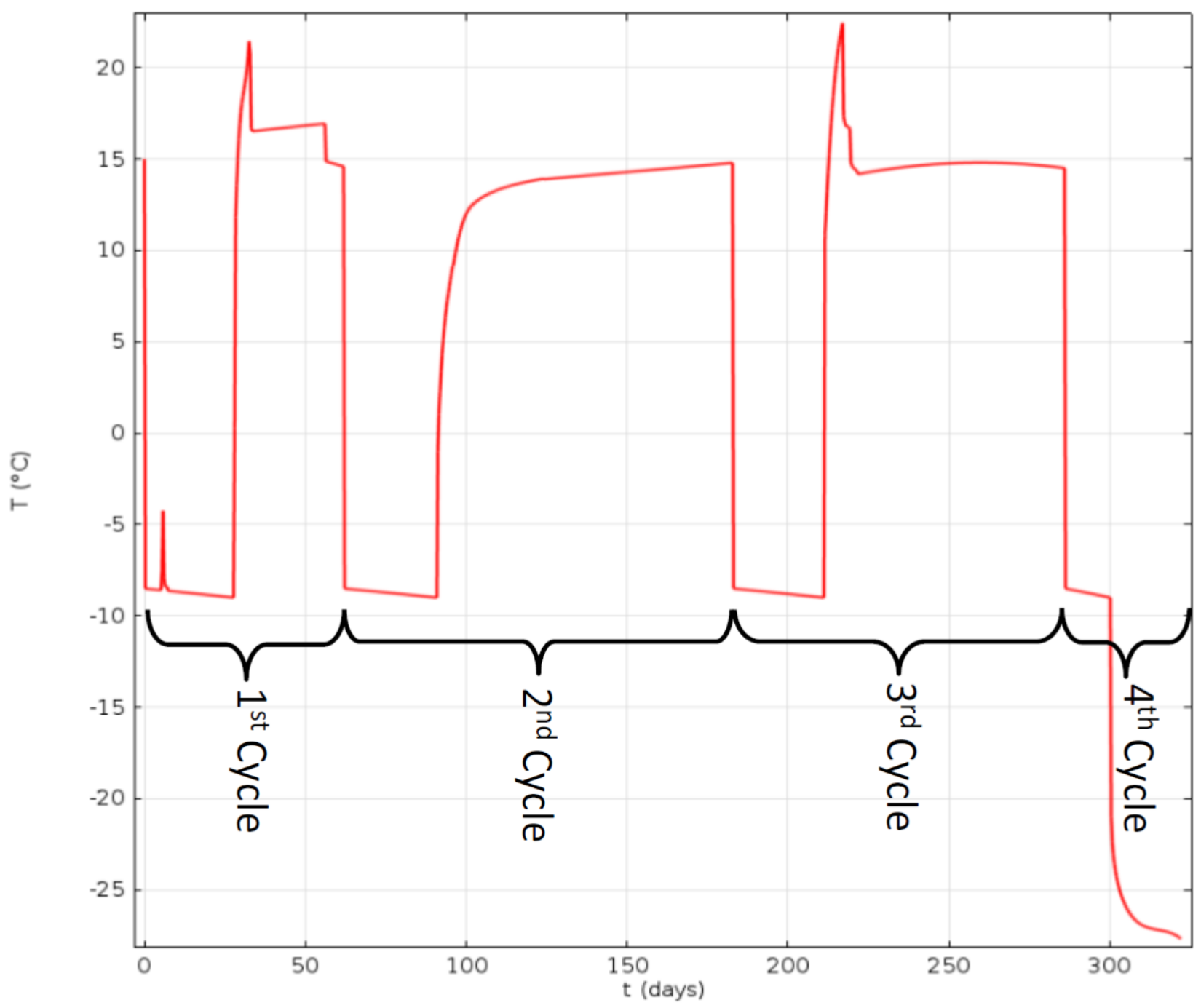


Fig. 3. Central multi-sensor ultrasonic probe being placed in the borehole (left), ultrasonic sensor fitted on the surface (top right) and ultrasonic probe shifted in place (bottom right).

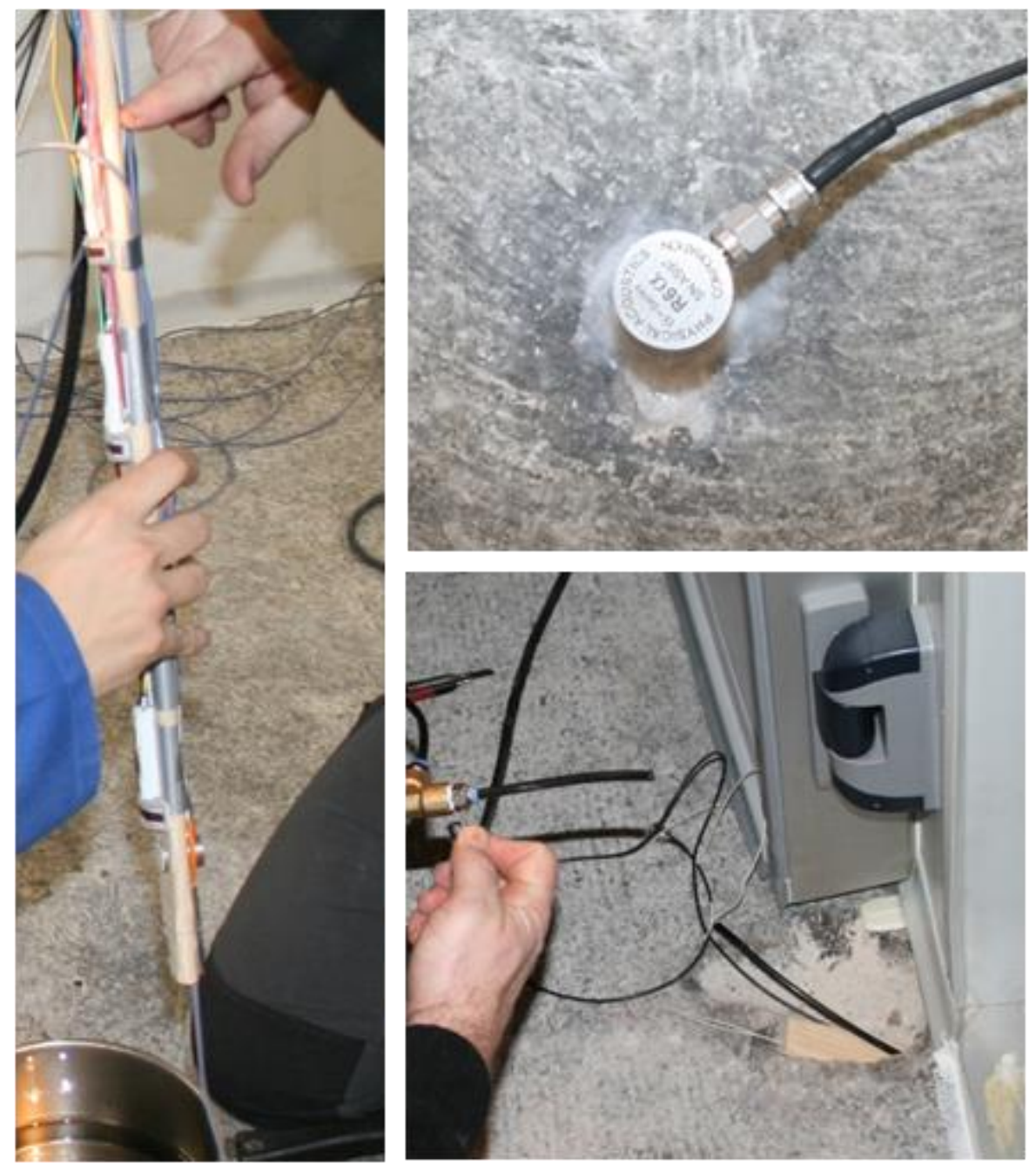


Fig. 4. Location of the ultrasonic sensors on a plane view (top) and vertical view (bottom).

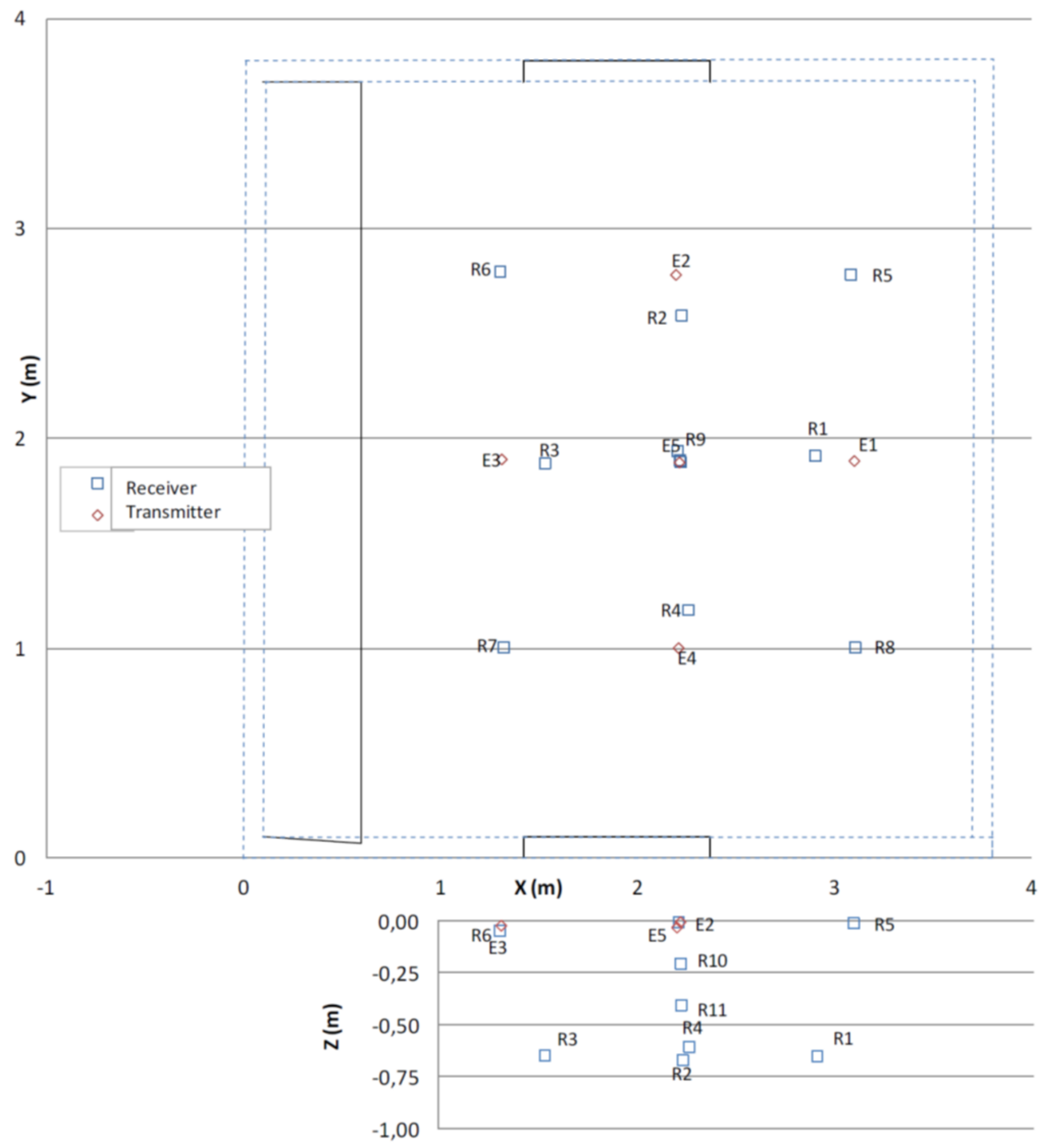


Fig. 5 Camera footage as the ball drops.

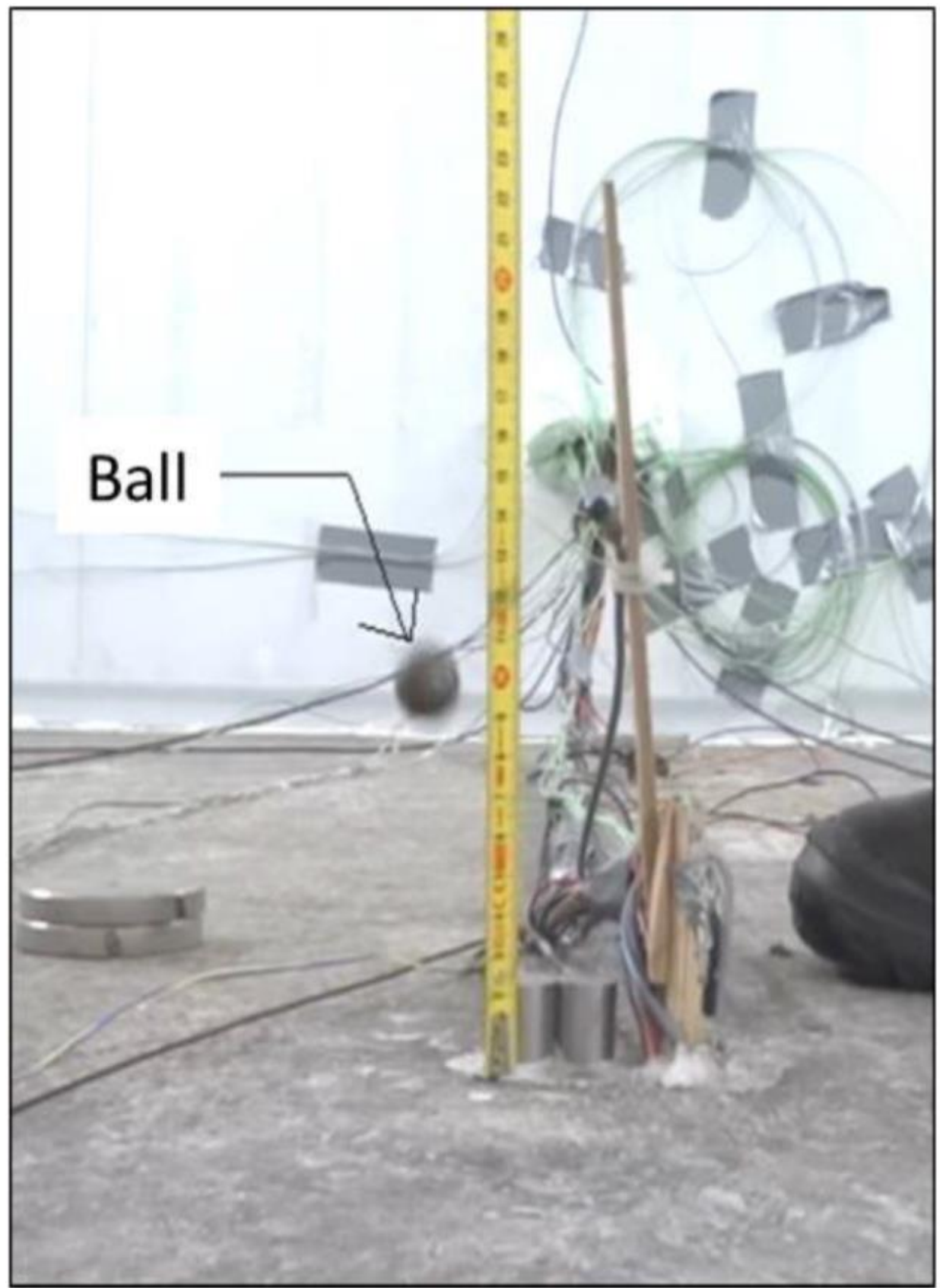


Fig. 6. Graph showing the proper correlation between the average energy values at source calculated for all sensors at each event and the theoretical energy values at source calculated from the drop and rebound heights.

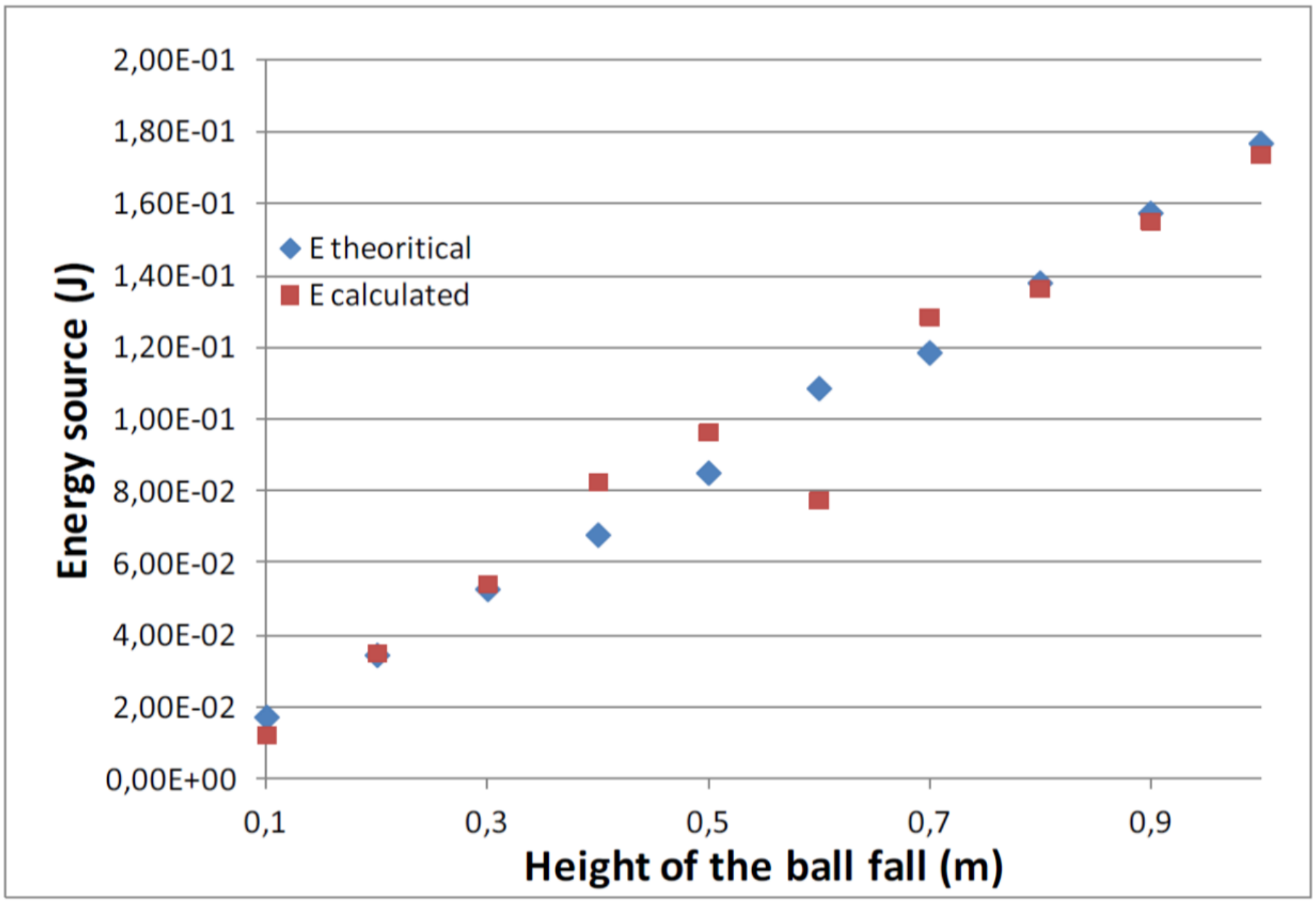


Fig. 7. Fracture mode corresponding to the acoustic signal and relationship between average frequency and RA value for norm acoustic emissions.
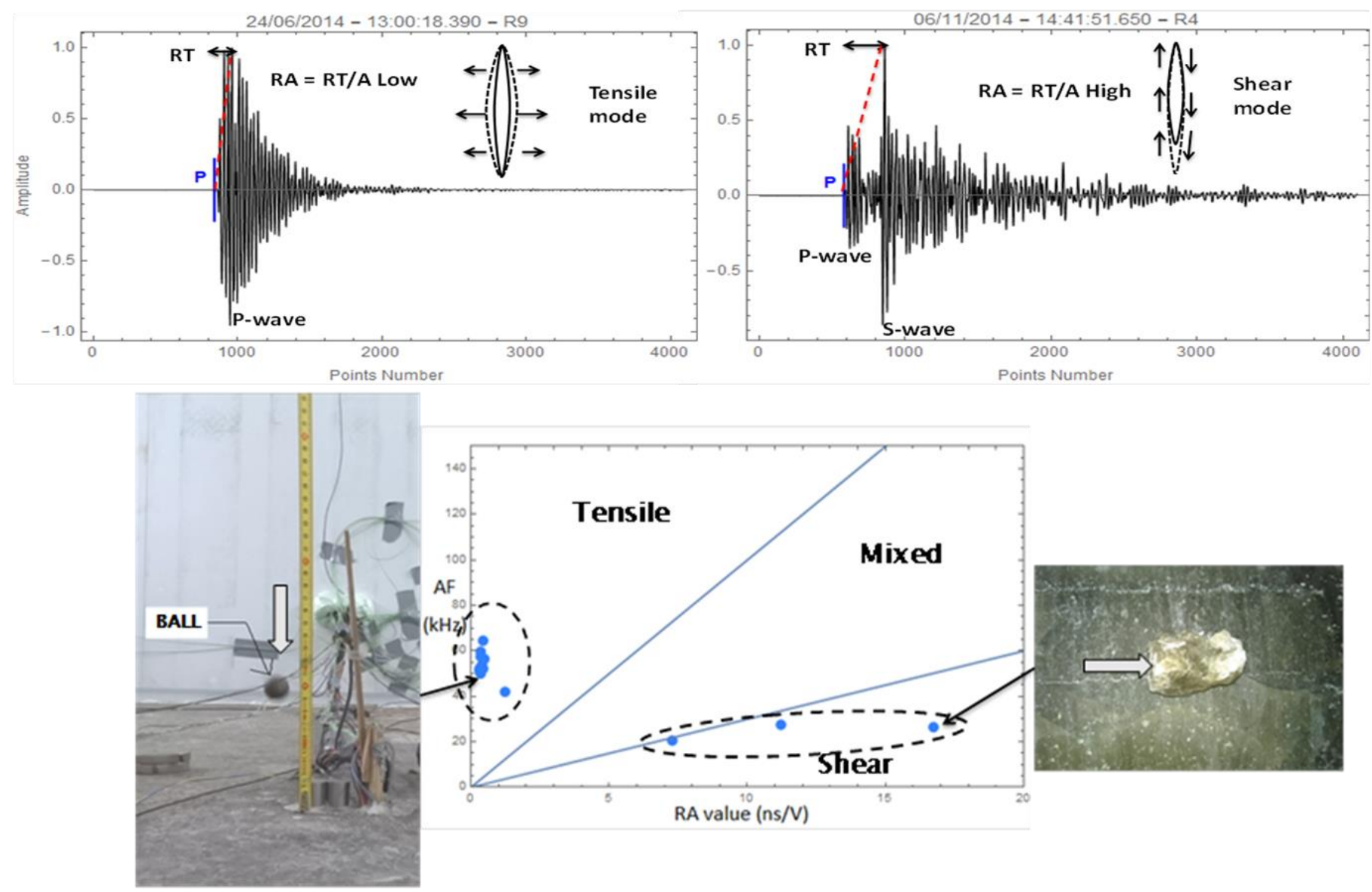
Fig. 8. Changes in the number of events following the cooling cycles.

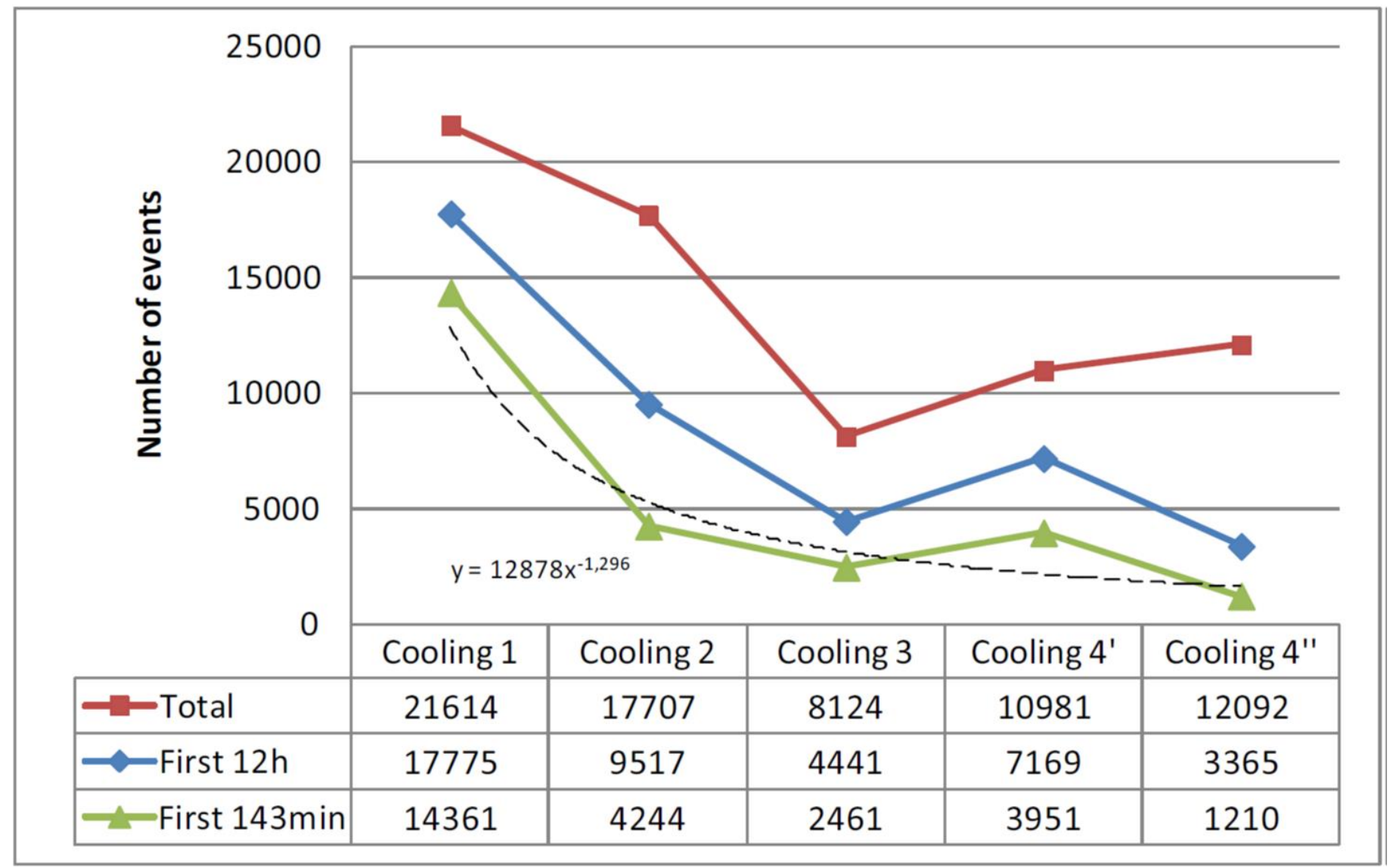


Fig. 9. Number of acoustic emissions during the 4 cooling cycles (cooling cycle $N^{\circ} 1$ : blue; cooling cycle $\mathrm{N}^{\circ} 2$ : red; cooling cycle $\mathrm{N}^{\circ} 3$ : Green; cooling cycle $\mathrm{N}^{\circ} 4$ (4' and 4"): grey) and zoom at the start ( 0.1 day or $143 \mathrm{~min}$ ). (For interpretation of the references to color in this figure legend, the reader is referred to the web version of this article.)
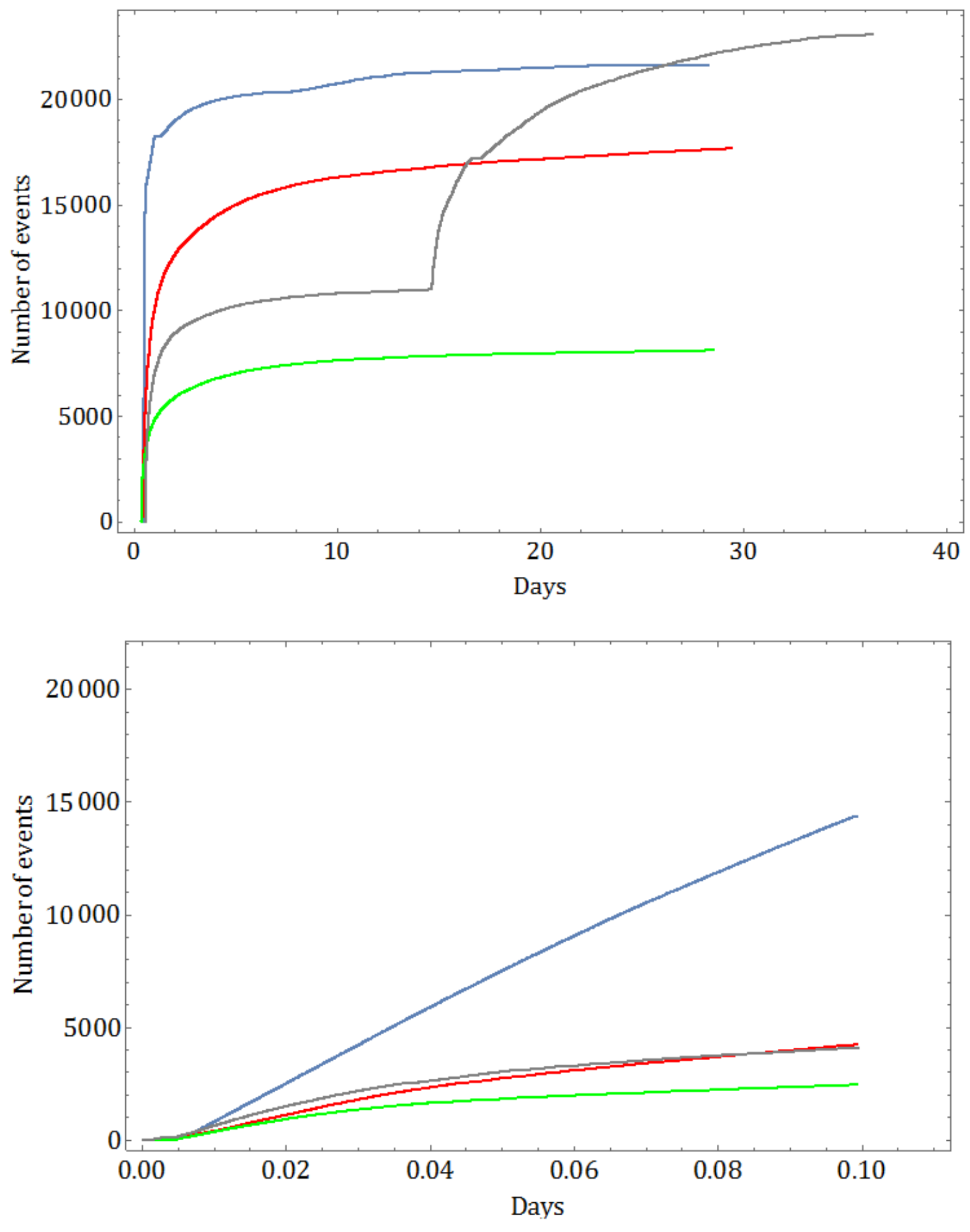
Fig. 10. Fluctuations in average and dominant magnitudes (the dominant magnitude is the magnitude most widely represented among all acoustic emissions) of the events following the cooling cycles.

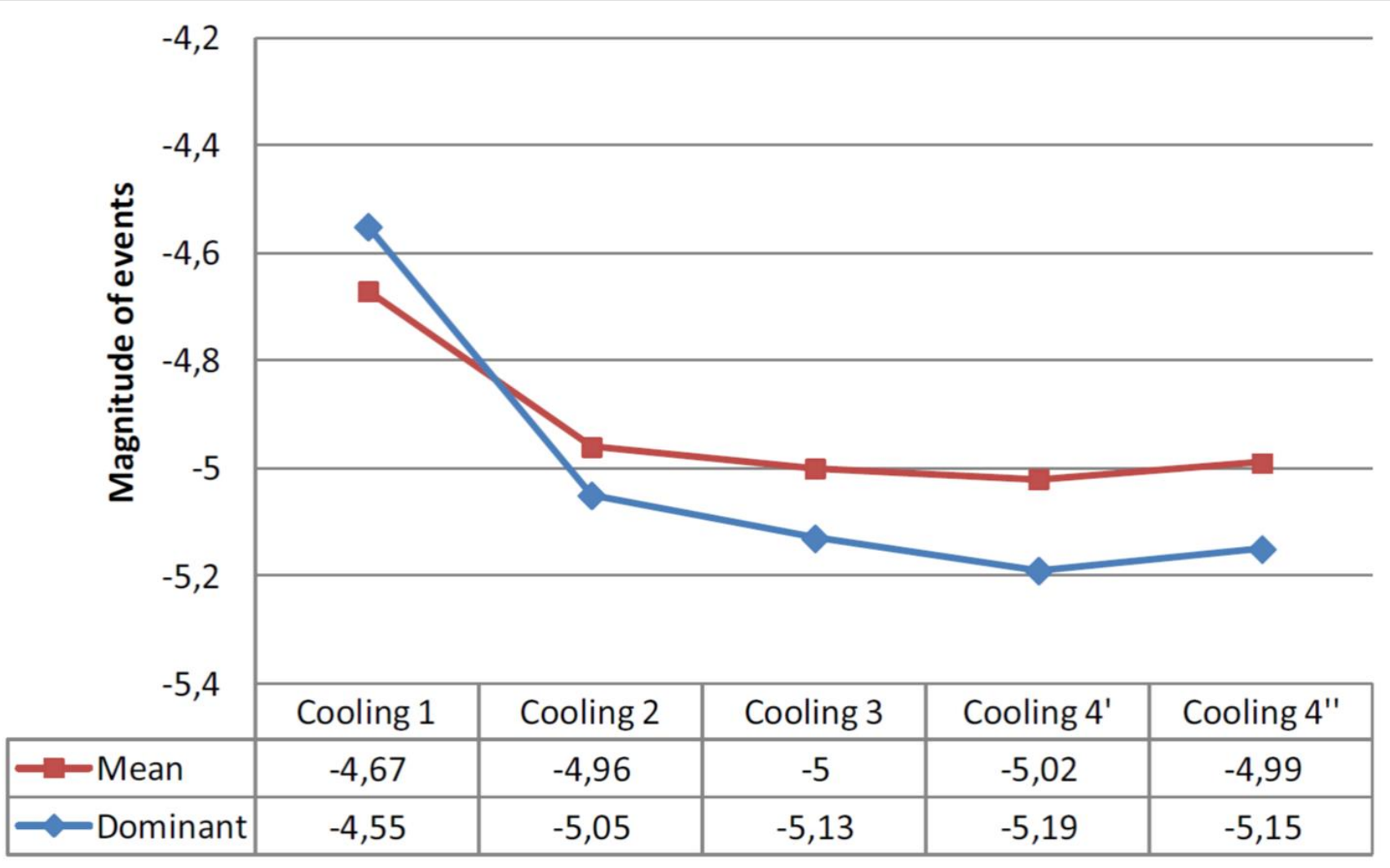

Fig. 11. b-value calculated for the 4 cooling regarding the depth of the AE.

$\operatorname{Depth}(\mathrm{m})$

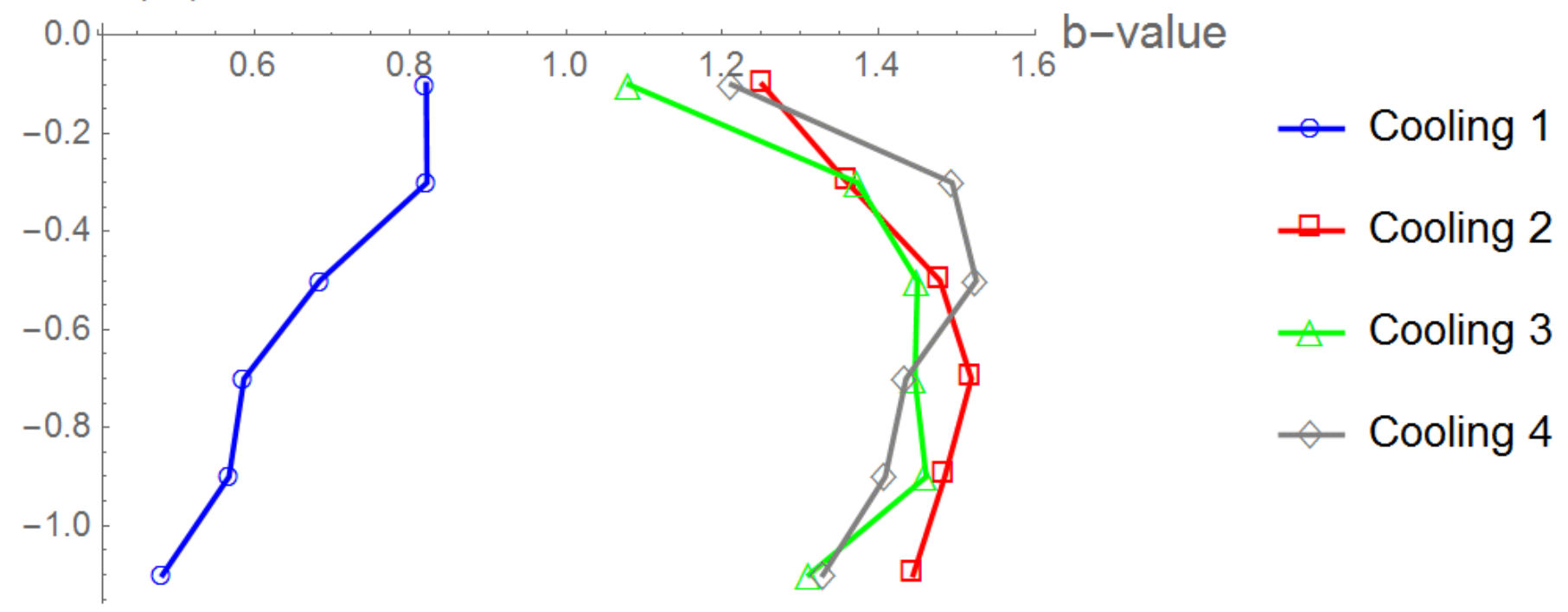


Fig. 12. Variation in depth of acoustic emissions during the 4 cooling cycles, up and right (cooling cycle $\mathrm{N}^{\circ}$ 1: blue; cooling cycle $\mathrm{N}^{\circ} 2$ : red; cooling cycle $\mathrm{N}^{\circ} 3$ : green; cooling cycle $\mathrm{N}^{\circ} 4$ (4' and $\left.4^{\prime \prime}\right)$ : grey); moving average values of 100 acoustic emissions. (For interpretation of the references to color in this figure legend, the reader is referred to the web version of this article.)

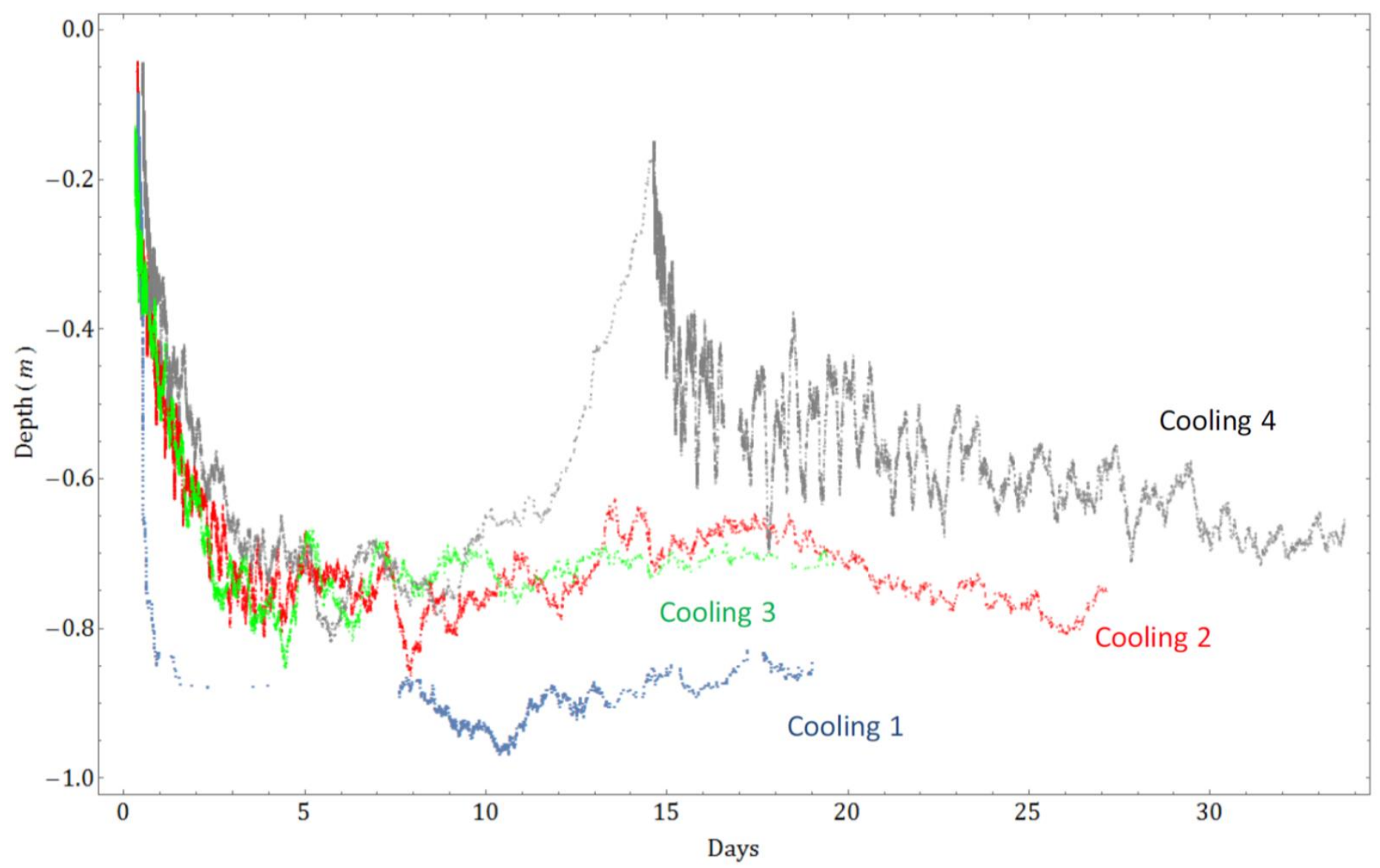

Fig. 13. Picture of cracks on the surface salt and specific radius of a -5 magnitude $A E$.

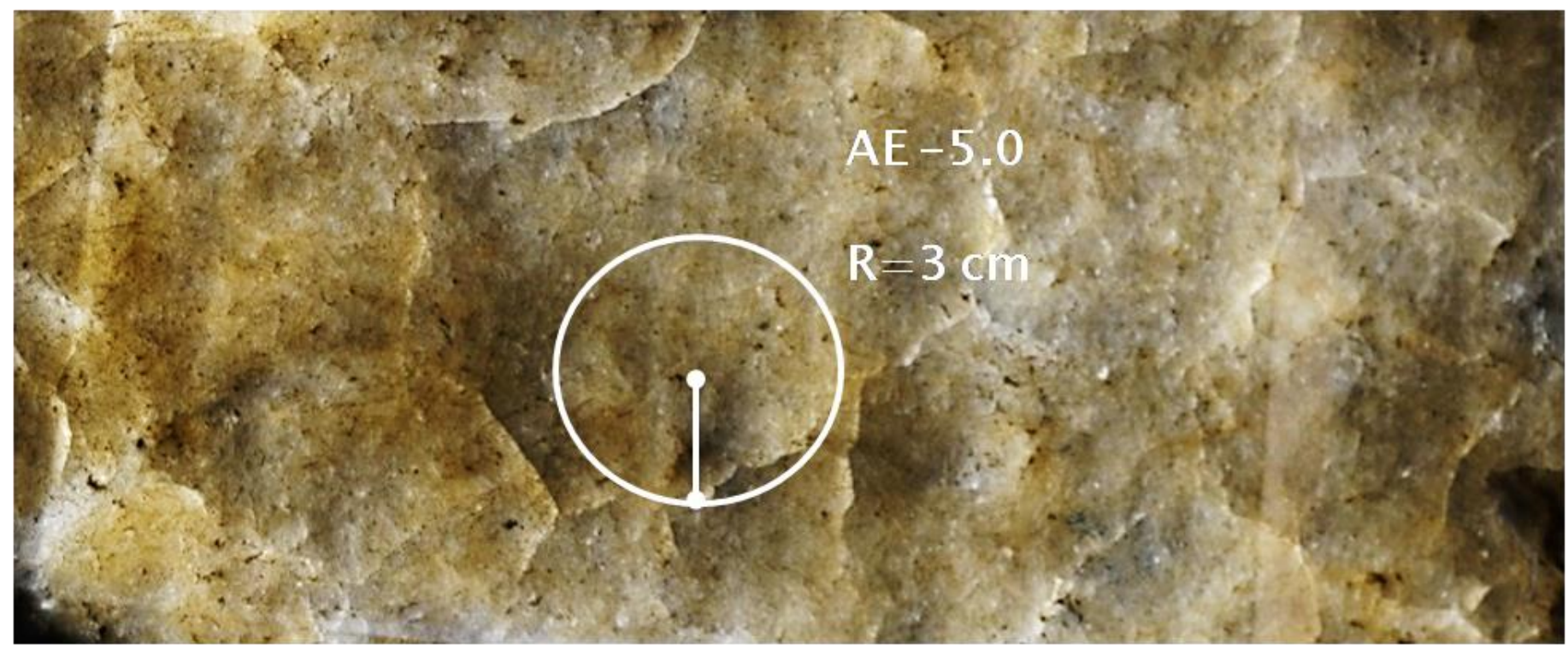


Fig. 14. Variations in the thermal gradient calculated between the borehole heat sensors (for cycle 2) and the depth variations of the acoustic emissions (moving average of 100 acoustic emissions).

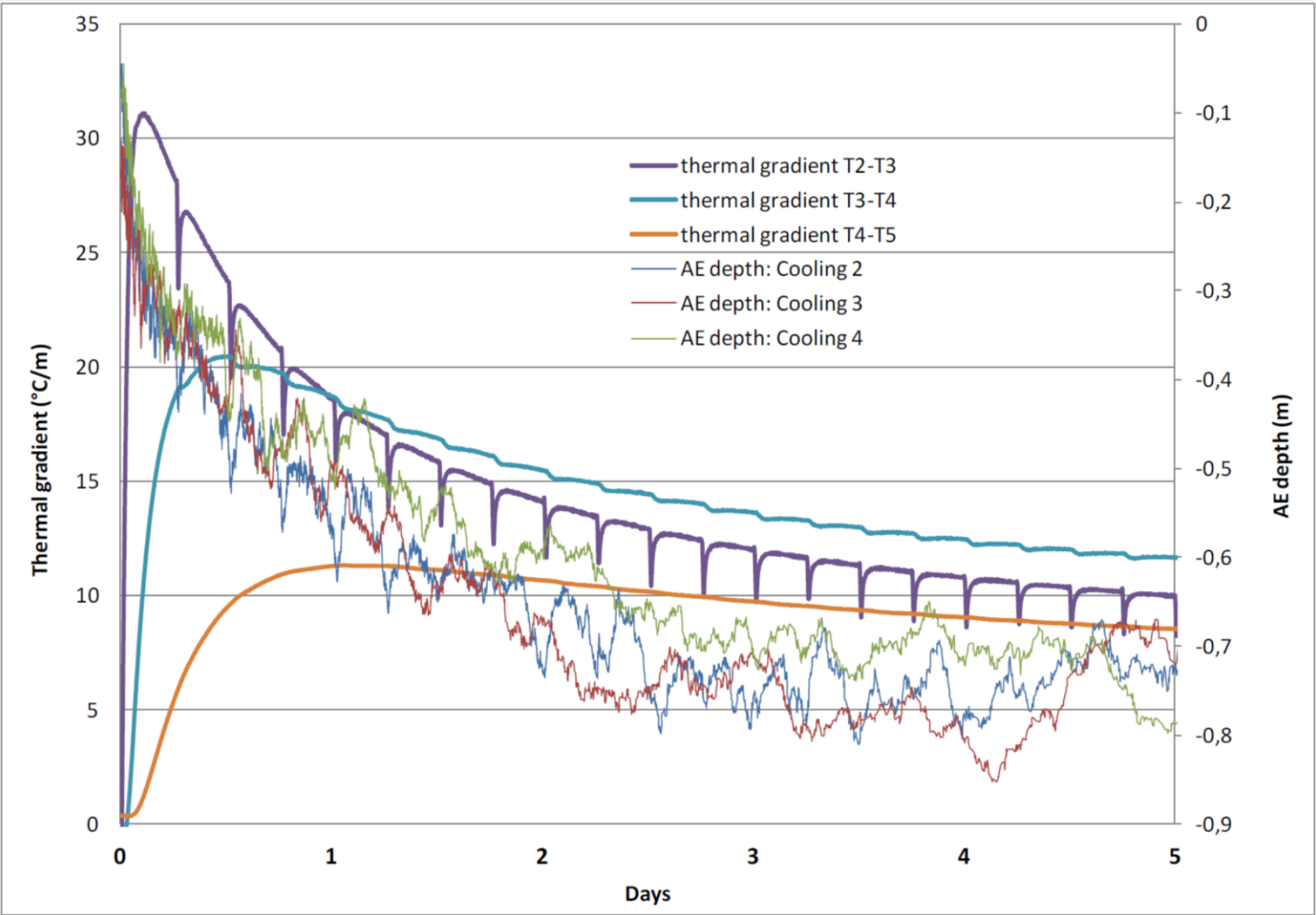


Fig. 15. Average frequency as a function of rise time for the first of the shallowest acoustic emissions (left) and the last, deeper acoustic emissions (right) in the 2nd cooling cycle (up) and in the 3rd cooling cycle (down).
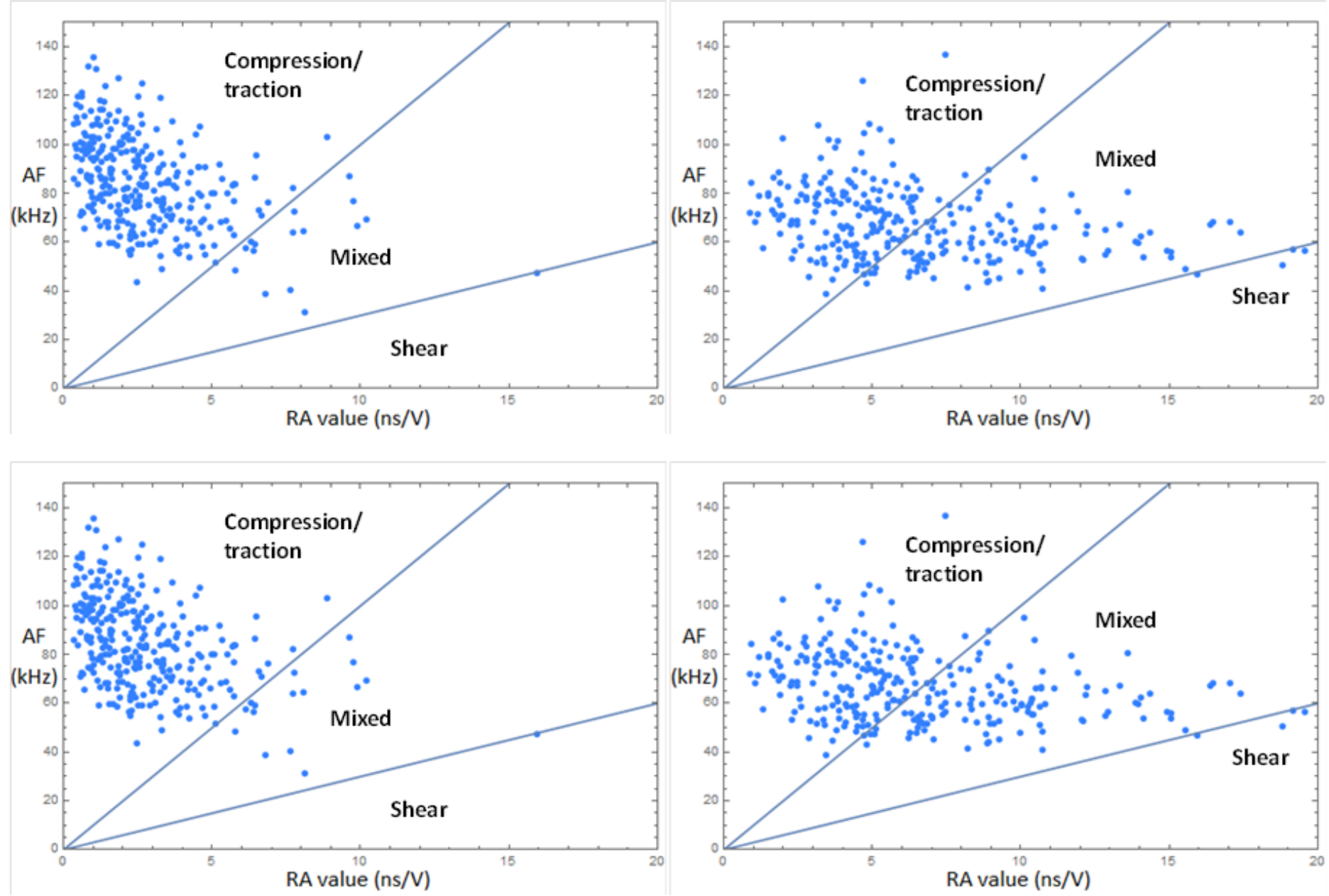

Fig. 16. Comparison between the macroscopic surface cracks (a) and the cumulated acoustic energy density versus time top view and cross section after $15 \mathrm{~min}$ (b), after $30 \mathrm{~min}$ (c) and after one hour (d).

Macroscopic

(a) Surface cracks $\begin{gathered}\text { Acoustic } \\ \text { sensor }\end{gathered}$

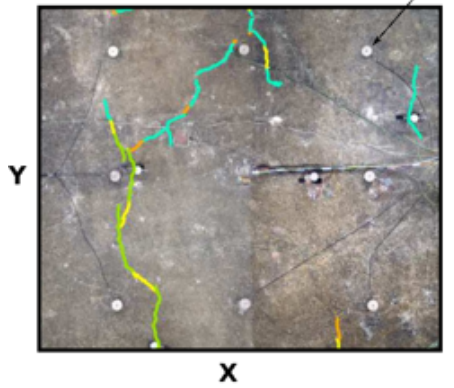

$\begin{array}{ll} & \text { 1st cooling } \\ 1 \mathrm{~m} & \text { 2nd cooling } \\ - & \text { 3rd coooling }\end{array}$
Acoustic emission of first cooling cycle

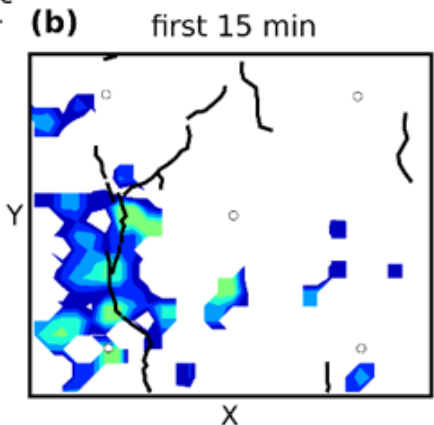

(c)

(d)
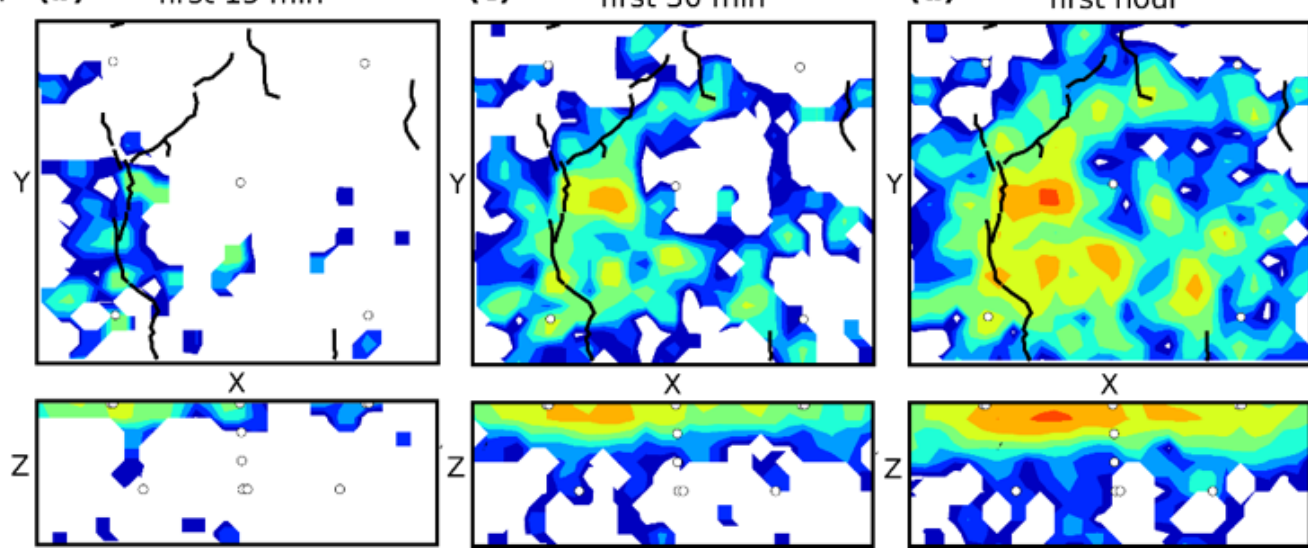
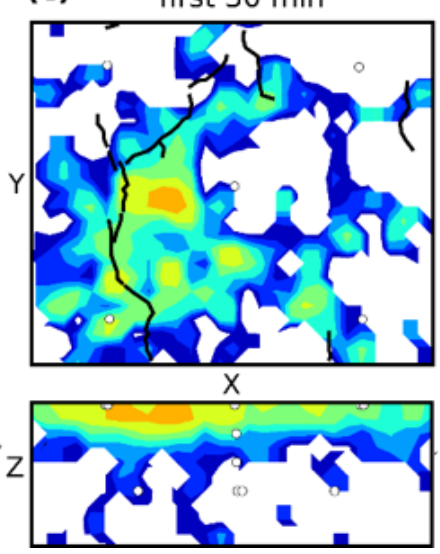

$\mathrm{X}$

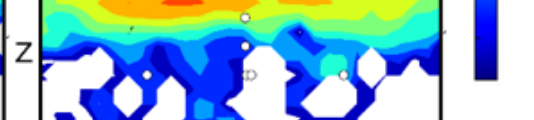

